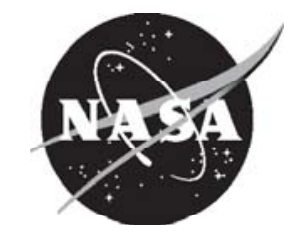

\title{
A Process for Assessing NASA's Capability in Aircraft Noise Prediction Technology
}

Milo D. Dahl

Glenn Research Center, Cleveland, Ohio 


\section{NASA STI Program . . . in Profile}

Since its founding, NASA has been dedicated to the advancement of aeronautics and space science. The NASA Scientific and Technical Information (STI) program plays a key part in helping NASA maintain this important role.

The NASA STI Program operates under the auspices of the Agency Chief Information Officer. It collects, organizes, provides for archiving, and disseminates NASA's STI. The NASA STI program provides access to the NASA Aeronautics and Space Database and its public interface, the NASA Technical Reports Server, thus providing one of the largest collections of aeronautical and space science STI in the world. Results are published in both non-NASA channels and by NASA in the NASA STI Report Series, which includes the following report types:

- TECHNICAL PUBLICATION. Reports of completed research or a major significant phase of research that present the results of NASA programs and include extensive data or theoretical analysis. Includes compilations of significant scientific and technical data and information deemed to be of continuing reference value. NASA counterpart of peer-reviewed formal professional papers but has less stringent limitations on manuscript length and extent of graphic presentations.

- TECHNICAL MEMORANDUM. Scientific and technical findings that are preliminary or of specialized interest, e.g., quick release reports, working papers, and bibliographies that contain minimal annotation. Does not contain extensive analysis.

- CONTRACTOR REPORT. Scientific and technical findings by NASA-sponsored contractors and grantees.

- CONFERENCE PUBLICATION. Collected papers from scientific and technical conferences, symposia, seminars, or other meetings sponsored or cosponsored by NASA.

- SPECIAL PUBLICATION. Scientific, technical, or historical information from NASA programs, projects, and missions, often concerned with subjects having substantial public interest.

- TECHNICAL TRANSLATION. Englishlanguage translations of foreign scientific and technical material pertinent to NASA's mission.

Specialized services also include creating custom thesauri, building customized databases, organizing and publishing research results.

For more information about the NASA STI program, see the following:

- Access the NASA STI program home page at http://www.sti.nasa.gov

- E-mail your question via the Internet to help@ sti.nasa.gov

- Fax your question to the NASA STI Help Desk at 301-621-0134

- Telephone the NASA STI Help Desk at 301-621-0390

- Write to: NASA Center for AeroSpace Information (CASI) 7115 Standard Drive Hanover, MD 21076-1320 


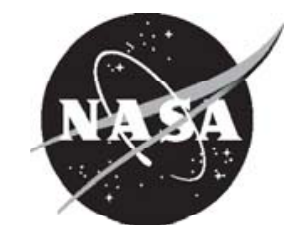

\section{A Process for Assessing NASA's Capability in Aircraft Noise Prediction Technology}

Milo D. Dahl

Glenn Research Center, Cleveland, Ohio

Prepared for the

14th Aeroacoustics Conference

sponsored by AIAA and CEAS

Vancouver, British Columbia, Canada, May 5-7, 2008

National Aeronautics and

Space Administration

Glenn Research Center

Cleveland, Ohio 44135 
This report contains preliminary findings, subject to revision as analysis proceeds.

This work was sponsored by the Fundamental Aeronautics Program at the NASA Glenn Research Center.

Level of Review: This material has been technically reviewed by technical management.

Available from

NASA Center for Aerospace Information 7115 Standard Drive

Hanover, MD 21076-1320
National Technical Information Service 5285 Port Royal Road Springfield, VA 22161

Available electronically at http://gltrs.grc.nasa.gov 


\title{
A Process for Assessing NASA's Capability in Aircraft Noise Prediction Technology
}

\author{
Milo D. Dahl \\ National Aeronautics and Space Administration \\ Glenn Research Center \\ Cleveland, Ohio 44135
}

\begin{abstract}
An acoustic assessment is being conducted by NASA that has been designed to assess the current state of the art in NASA's capability to predict aircraft related noise and to establish baselines for gauging future progress in the field. The process for determining NASA's current capabilities includes quantifying the differences between noise predictions and measurements of noise from experimental tests. The computed noise predictions are being obtained from semi-empirical, analytical, statistical, and numerical codes. In addition, errors and uncertainties are being identified and quantified both in the predictions and in the measured data to further enhance the credibility of the assessment. The content of this paper contains preliminary results, since the assessment project has not been fully completed, based on the contributions of many researchers and shows a select sample of the types of results obtained regarding the prediction of aircraft noise at both the system and component levels. The system level results are for engines and aircraft. The component level results are for fan broadband noise, for jet noise from a variety of nozzles, and for airframe noise from flaps and landing gear parts. There are also sample results for sound attenuation in lined ducts with flow and the behavior of acoustic lining in ducts.
\end{abstract}

\section{Preface}

The assessment of NASA's capability in aircraft noise prediction is a process that, at the time of writing this paper, is still undergoing analysis and review. It is NASA's intent at the end of this process to publish a complete documentation of the results. This paper describes the process of the assessment and illustrates the types of results generated. The project was divided into topic areas each with multiple contributors including airframe noise (J. H. Casper, D. P. Lockard, C. L. Burley, M. M. Choudhari, W. M. Humphreys, F. V. Hutcheson, M. R. Khorrami, and C. L. Streett), aircraft systems (C. L. Burley, J. W. Rawls Jr., J. J. Berton, and M. A. Marcolini), engine systems (L. S. Hultgren, J. H. Miles, and P. C. E. Jorgenson), jet noise (J. E. Bridges, A. Khavaran, and C. A. Hunter), fan noise (E. Envia, D. L. Tweedt, D. M. Elliott, E. B. Fite, C. E. Hughes, G. G. Podboy, D. L. Sutliff, and R. P. Woodward), propulsion airframe aeroacoustics (R. H. Thomas and C. A. Hunter), duct acoustics (W. R. Watson, D. M. Nark, M. G. Jones, and T. L. Parrott), and liner physics (T. L. Parrott, M. G. Jones, W. R. Watson, C. H. Gerhold, and M. C. Brown). The sample results section of this paper is based on contributions from these researchers and the results should be considered preliminary pending review and publication of the final NASA assessment report.

\section{Introduction}

The NASA Fundamental Aeronautics Program under the Aeronautics Research Mission Directorate has as part of its vision the development of physics-based multi-disciplinary design, analysis, and optimization (MDAO) tools. Currently, most computer-based tools that may be applied as MDAO-like tools are highly customized to specific configurations and processes. A change to the configuration or process usually requires extensive and complex redevelopment efforts to achieve new modeling solutions. These can range from analysis tools that are empirical in nature and do not handle unconventional geometries well without new experimental data, redesign, and recalibration to highly intensive computational methods that take specialists 
to operate and take inordinate amounts of time to obtain a single solution set. The challenge is to create a tool system that has flexibility in the fidelity of the calculation and to enable the study of the flight envelope and design space of new aircraft that may be outside of current convention. To achieve this vision, NASA needs robust, highly accurate tools and methods for performance prediction. These tools require quantified levels of uncertainty and need to be validated with experimental data that also include established uncertainty levels. This emphasis on quantifying uncertainty is meant to better focus the effort and to increase the credibility of the results. The common method of "graphical validation" is inadequate and the quantification of error and uncertainty is a must. ${ }^{1}$ The Fundamental Aeronautics Program contains four projects covering subsonic, supersonic, and hypersonic aircraft as well as subsonic rotorcraft. Each project is structured with a base of foundational research to provide knowledge for various disciplines to develop tools and technologies at the component level. These components will be integrated into subsystems and finally into full systems each with appropriate testing and validation programs. The MDAO capability will be a product of this development process. Among the disciplines defined in the program, the acoustics discipline focuses on developing improved noise prediction methods, at both the fundamental and component level, and on developing technologies to lower the noise radiated from the aircraft. At the start of this program, NASA undertook an assessment of its current capabilities to predict noise from aircraft systems and components. This paper describes the process used in this assessment and uses highlights and sample results to illustrate the various types of results obtained when predictions of aircraft noise, at both the system and component levels, are compared to measurement data.

The current capability for system level aircraft noise prediction is represented by tools such as NASA's Aircraft Noise Prediction Program (ANOPP). ${ }^{2}$ The ANOPP code, initially developed in the early 1970's, ${ }^{3,4}$ assembles source prediction modules for engine and airframe components and links them to an acoustic propagation code to obtain far field noise levels and sound spectra. In addition, the combined noise source representation of an aircraft can be moved in time along simulated trajectories to obtain noise levels for various observers along or near this simulated flight path. Various modules in ANOPP have been updated over the years as new data sets became available; however, the empirical nature of the program limits its usefulness. ANOPP results typically show large discrepancies when attempting to predict radiated noise for aircraft designs outside of its database. Even with this limitation, ANOPP, in its current form, represents the state of the art in the ability to do rapid prediction estimates of aircraft system level noise.

The computational approaches to predicting aircraft noise that rely more on using first-principal equations than on empirical source modeling, especially at the component level, use increasingly higher fidelity codes such as those based on analytical, statistical, and numerical methods. Analytical methods use a rearrangement of the governing equations in a manner following the acoustic analogy approach. The resulting equation contains a linear wave operator on one side of the equation and a source term on the other side. For fan noise generation, the acoustic source is the unsteady pressure distribution generated on the surface of a blade when a vortical disturbance velocity field interacts with the blade surface. ${ }^{5}$ This aerodynamic source may be obtained by measurements, through computations, or by using a model. The acoustic solution is obtained by first computing the acoustic source model followed by solving an integral equation for the acoustic field. Depending how the solution is structured, one can, for example, compute the blade-passage-frequency tones associated with rotor-stator interaction ${ }^{6}$ or compute the fan broadband noise generated by the interaction of the fan rotor turbulent wake with the downstream stator vanes. ${ }^{7}$ The statistical approaches start with a similar rearrangement of the governing equations into an equation with a linear wave operator on one side and source terms on the other side. However, in this case, the sources are spatially distributed and identified with the turbulent fluctuations in the flow field. Computational fluid dynamics (CFD) calculations using steady Reynolds-Averaged Navier-Stokes (RANS) codes with turbulence models are now used to provide integral length and time scales that affect the functional descriptions characterizing the statistical behavior of the sources. ${ }^{8}$ Finally, numerical methods solve the unsteady noise generation problem directly from the governing equations, ${ }^{9}$ or solve the propagation problem in complex geometries given a known source. ${ }^{10}$ Presently, computational capabilities limit the unsteady solution to the source region. To obtain the far field radiated noise, techniques such as the Kirchhoff or the Ffowcs Williams-Hawkings methods are used to propagate the sound outward from near the source region. ${ }^{11}$

A specific listing of the prediction codes used in this assessment is shown in Table 1. This listing does not contain all possible codes within NASA that relate to aircraft noise prediction; nor does it contain codes external to NASA in other government, industry, or academic organizations that may be a similar type of code or a variation of these codes. Due to limitations of time and resources, the codes shown here were 


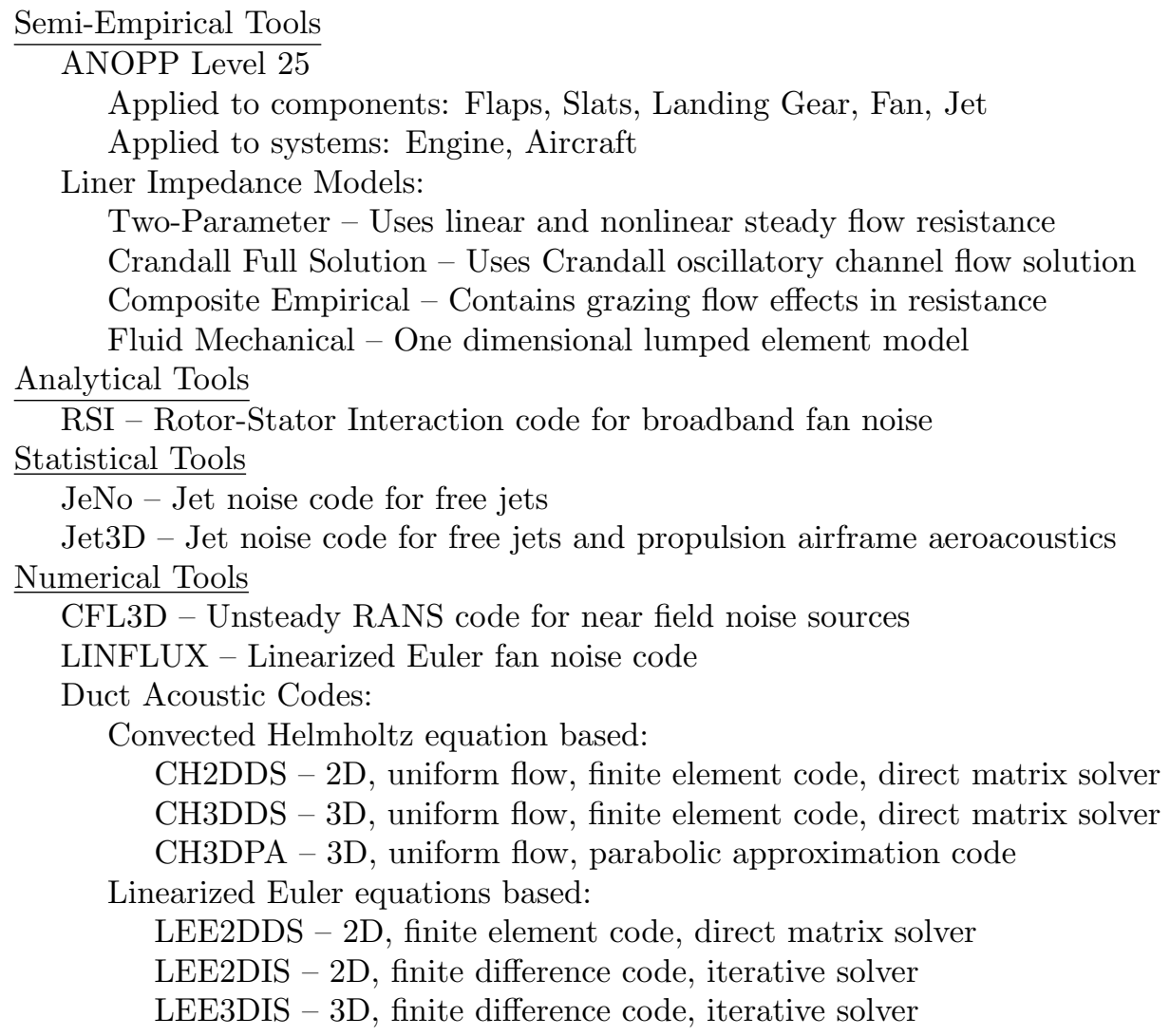

Table 1. List of codes assessed for aircraft noise predictive capability.

chosen as representative of the state-of-the-art or current capability in aircraft noise prediction at NASA. Many codes requiring intense computational resources such as codes using computational aeroacoustics and large eddy simulation techniques are considered to be part of on-going research and thus not part of this assessment of aircraft noise prediction capability.

In the next section, the process of the assessment is described and terms are defined. Validation is a term often used to denote the ability of computed results to agree with experimental data. This is recognized as a continual process as more data becomes available leading to perhaps a requirement to make code modifications leading to more validation. In this context, assessment is a snapshot in time of the current state of a validation process. At all times in this process, data to quantify the errors and uncertainties are gathered to document progress in prediction capability. This is followed by a section showing sample assessment results that include estimates of errors and uncertainties. Since this assessment process is not yet complete, these results are being used to illustrate the various types of results generated as a result of this assessment and should be considered preliminary pending final review and publication. All the data and all the results will be documented in detail in a NASA Technical Publication.

\section{Assessment Process}

The assessment of NASA's current capability to predict aircraft noise is based on quantifying the differences between predictions of noise from computer codes and measurements of noise from experimental tests. The credibility of this comparison is further enhanced by quantifying the uncertainty or accuracy of both the computer predicted results and the experimental measured results. For the computed results, there are no methodologies for determining uncertainties that are unique to the computer codes related to aircraft noise prediction. These codes are built using computing practices typical of their empirical, analytical, statistical, or numerical basis. Hence, we have followed well documented practices that are found in the literature, ${ }^{1,12}$ in technical guides, ${ }^{13}$ and in engineering references ${ }^{14,15}$ for determining computational uncertainties and for 
determining experimental uncertainties. The literature related to computer modeling and simulation validation is full of the usage of terms like verification, validation, uncertainty, and error. Some of these terms are used interchangeably. The AIAA guide (G-077-1998) ${ }^{13}$ on validating CFD simulations states that a failure to distinguish between these terms reduces the credibility in validating computer modeling and simulation. To promote clarity and uniformity in performing this assessment of aircraft noise prediction codes, we adopted definitions for the terms given below that follow precedence in the cited literature.

1. Verification is the process of determining that a model is implemented accurately. It includes ensuring that the controlling physical equations have been correctly implemented in a computer code, finding and removing mistakes and errors in the numerical algorithm and in the code, making sure that the input data is accurate, and estimating the numerical solution error, as appropriate, through convergence studies.

2. Validation can be defined as the evidence which demonstrates that the computer model is correct by comparing computer results with experimental data. The experimental data may be obtained from a variety of tests of differing complexity including from fundamental experiments, from sub-system tests, and from complete system tests. However, it should be recognized that the complete correctness or accuracy of the computer model to all possible solutions cannot be proven. Hence, validation is considered a process of determining accuracy as evidence from real world experiments is continually gathered, which may in turn require modifications to the computer model, and more validation, etc.

3. Uncertainty is part of many aspects in obtaining the computed results and the experimental data. In a general definition, the AIAA guide suggests that uncertainty is a potential deficiency in any part of the computer modeling and the experimental data gathering process that is due to a lack of knowledge. Definitions for different types of uncertainties are given to help clarify the type of uncertainty being computed, for example:

Numerical Uncertainty: The uncertainty in computed results due to grid size, time steps, number of iteration, etc., that affect computationally-based methods more than empirical methods. These uncertainties are typically obtained through code verification and convergence studies. Numerical uncertainty is assumed to be uncorrelated with model uncertainty and, if it is significant, may be combined with model uncertainty.

Model Uncertainty: The uncertainty in computed results due to initial conditions, boundary conditions, and model parameters. A deficiency or incomplete knowledge of a physical parameter, for instance, is often represented by a probability function. Thus, a statistical process is essentially used to determine computed uncertainty. Estimates of the uncertainty of the code output can be obtained from variations in these inputs to the code using sensitivity analysis and uncertainty analysis. Computationally-based codes are assumed to be converged for this type of error analysis.

(a) Sensitivity Analysis: Codes may potentially have a large number of input parameters. A sensitivity analysis is performed to reduce the number of important parameters. This analysis reveals which input parameters most affect the output results. For each parameter:

i. Determine the range a parameter may have from experimental data or best estimate.

ii. Perform computations to determine the output effects for the input range of this parameter while all other input parameters are held constant.

(b) Uncertainty Analysis: Once the important input parameters are determined, the following procedure will determine the uncertainty of code output given the uncertainty in the input parameters:

i. Characterize input parameter uncertainty using experimental data or best estimate. Essentially, the input parameters are described by a probability density function with mean and variance values.

ii. Generate a series of input values using statistical sampling methods.

iii. Each sample set of input parameters is used in a computation to create an ensemble set of output values.

iv. Analyze the set of code outputs to quantify the uncertainty of the computed output. Realize that the accuracy of this analysis improves as the size of the sample set, and hence the number of computational runs, increases. 
A similar process was followed for experimental results. However, it has been recognized that there is no unique methodology for determining experimental uncertainty given the variability in the types of experimental data used for this assessment. Data came from laboratory tests, wind tunnel tests, and flight vehicle tests. Appropriate estimates of the experimental uncertainty depended on the particular situation and is discussed in the complete documentation of the assessment results.

4. The AIAA guide defines error as a recognizable deficiency in modeling that is not due to a lack of knowledge. The error may be categorized as acknowledged and unacknowledged. Truncation error when discretizing equations and physical approximations to simplify equations are examples of acknowledged error. Blunders and mistakes are considered to be some of the unacknowledged errors. There are many other errors that one may identify with an appropriate descriptive adjective. For the assessment task, the following general definition was used:

Error: The difference between the experimental measurement and the computed result.

Implicit in this definition is that both the experimental measurements and the computed results contain errors. Thus, the defined error is a resultant of all the errors that are associated with both. Ultimately, the effect of this error is to show how good or bad is the computer model. Given the uncertainties associated with both computations and experiments, if the error is much larger than the combined uncertainties, then the error is considered to be dominated by modeling error, assuming that what you measured in the experiment is actually what you were trying to model. This definition of error applies to a single comparison; but it also may apply to a series of similar comparisons. Hence, on average the error is some value with, of course, the appropriate combining of uncertainties included.

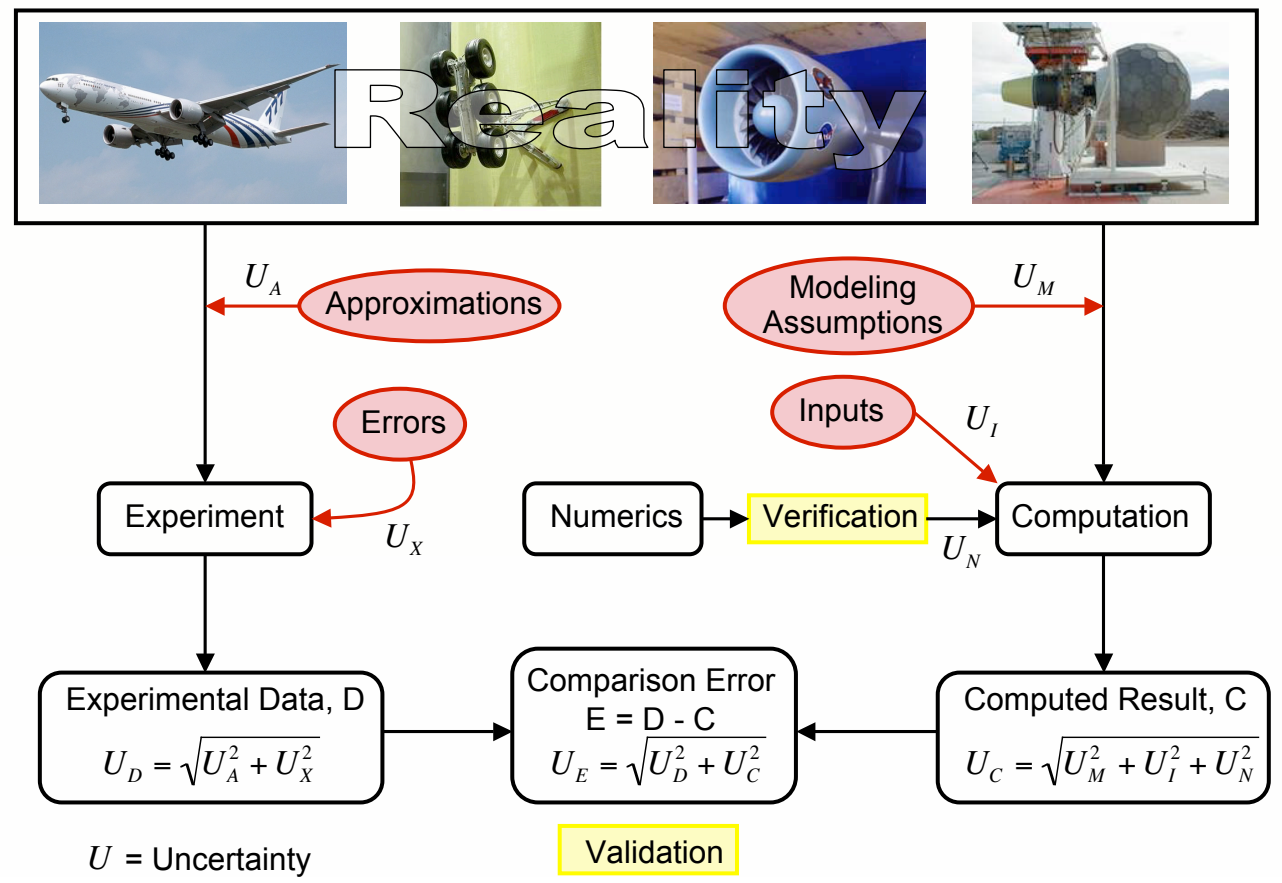

Figure 1. Diagram of an assessment process leading to the validation of a computed result by comparison to experimental data.

This process is illustrated in Figure $1 .^{\mathrm{a}}$ Reality is expressed by a particular component or system test configuration on which an experiment is to be conducted and for which a computer prediction is to be run. On the experimental side, certain approximations may be made as to what is being measured and errors may

${ }^{\text {a}}$ Figure 1 is based on a figure given in a presentation by H. W. Coleman at the Foundations for Verification and Validation in the 21st Century Workshop, John Hopkins University, Laurel, MD, October 22-23, 2002. 
be made during the experiment. For both, uncertainties exist, represented by a value $U$. The uncertainties are combined to obtain the total uncertainty associated with the experimental data.

Uncertainties are also found on the computational side including those associated with modeling assumptions, code inputs, and verification of the code numerical process. Modeling of the physics requires the development of mathematical equations to describe the physical processes which are thought to occur. In general, simplifications are made to obtain a tractable formulation. For example, complex three-dimensional geometries may be reduced to one- or two-dimensional approximations. In other cases, empirical correlations may be used to represent the physical process and enable the problem to be solved, sometimes rapidly. Computer codes require input parameters to run including initial and boundary conditions and model parameters internal to the code. Modeling and input uncertainties propagate through the code to create output uncertainties. In addition for more computationally-based codes, numerical uncertainties are defined through the verification process related to grid size, time step, iterations, etc., and are included in the total computational uncertainty.
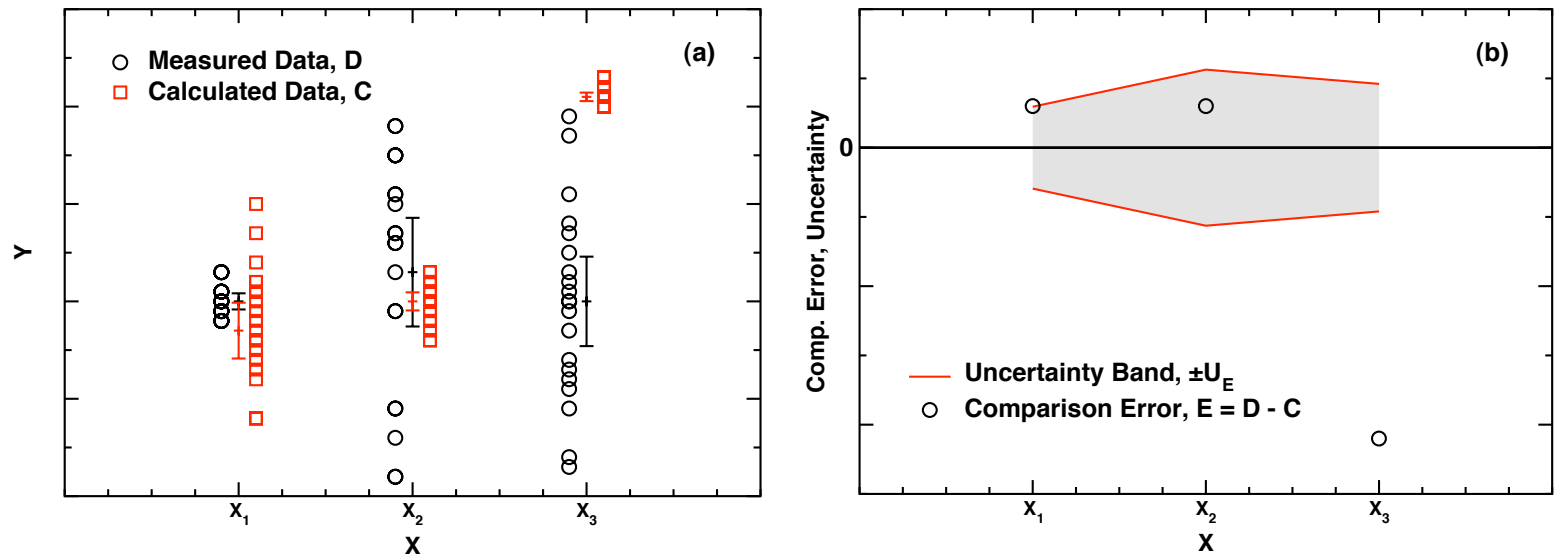

Figure 2. Example of determining errors and uncertainties using a statistical approach. (a) Raw measured and calculated data with computed means and $95 \%$ confidence intervals. (b) Resulting comparison error and uncertainty band.

As indicated in Figure 1, the final comparison error is determined from the difference between the experimental and computed results and the uncertainty associated with the comparison error is computed using the uncertainties from both the experimental and computed results. To further illustrate and explain the results computed by this assessment process, we turn to the sample results shown in Figure 2. Results are shown in Figure 2a for multiple measurements of a dependent variable $Y$ at three settings for the independent parameter $X$ along with multiple calculated predictions for the same parameter. There are 20 measurement points for each $X$ parameter. For the computations, the inputs and modeling parameters were randomly varied in 21 separate code runs to provide the calculated results. These raw data points are shown plotted on the figure slightly offset from their $X$-parameter value to better visualize the results. The mean values for these data and their $95 \%$ confidence intervals are shown as the small bars on the vertical lines. The confidence intervals indicate that the mean value has a $95 \%$ chance of being within the confidence interval given that the variance of the sample is a good estimate of the true variance value. From these results, we now have the experimental results $D$ and the computed results $C$ based on the mean values and their associated uncertainties $U_{D}$ and $U_{C}$ based on the $95 \%$ confidence intervals. The results of combining these data to obtain the comparison error and the combined uncertainty band are shown in Figure 2b. This plot illustrates the level of validation error within a quantified level of uncertainty. For parameter $X_{1}$, the comparison error lies just outside the uncertainty band. For parameter $X_{2}$, the comparison error lies within the uncertainty band and is perhaps indicative of a 'good' prediction compared to the result for parameter $X_{3}$ where the comparison error lies well outside of the uncertainty band indicating that either the computer model is wrong or there is something wrong in the experimental measurement process. However, further explanation is required before judgment is made as to the goodness of this example assessment. The comparison error results at parameters $X_{1}$ and $X_{2}$ may be considered borderline or good, respectively, if the uncertainty band is judged to be relatively small. Then any comparison error result that lies within the uncertainty band is considered equally valid given the current known level of uncertainty in the experimentally measured data 


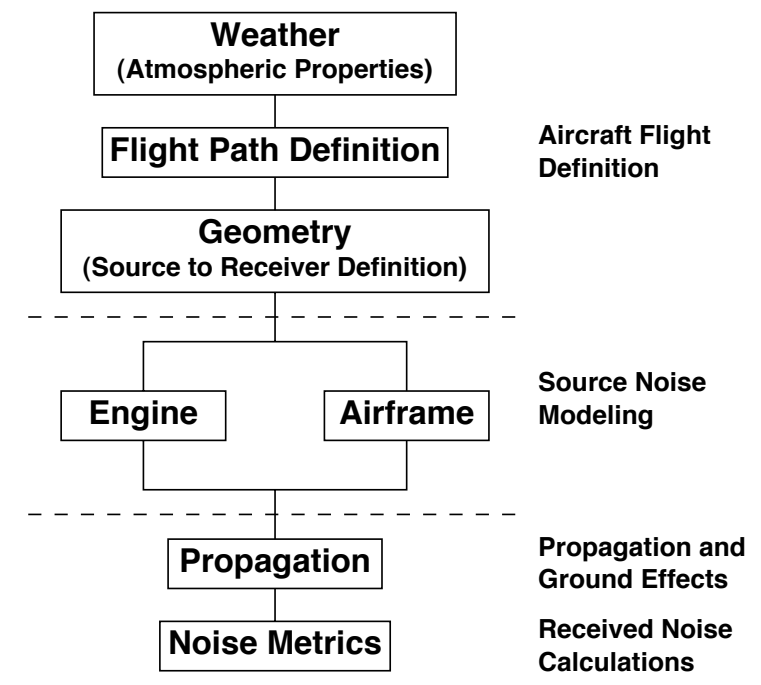

Figure 3. Schematic diagram of the structure of the ANOPP code.

and in the computed results. Conversely, if the uncertainty band is judged to be large, then neither results for the $X_{1}$ and $X_{2}$ cases can be considered a good validation of the predictive capability. The results for the $X_{3}$ parameter may always be regarded as a poor validation since the error is much larger than the uncertainty.

\section{Sample Results}

The assessment of NASA's capability to predict aircraft noise at the system level and at the component level includes both the comparison of predicted results with measured data and the quantification of errors and uncertainties. To illustrate the various types of results obtained, sample results from the assessment are used. First, the semi-empirical tools are considered where the modules of the ANOPP code are used to make component noise level predictions for fans, jets, and flaps. The types of results vary from the quality of results at a single operating condition, to comparing results over a large set of conditions, and to different results at model scale compared to full scale. The ANOPP code is also used to make predictions for engine systems and aircraft systems where module outputs are combined together. Next, computational codes of various forms are used to make component level noise predictions for a fan, jets, and a prototype landing gear component. Finally, the computer code approaches used to predict attenuation of sound in a flow duct and the properties of acoustic lining material provide a good example of using statistical uncertainty analysis for both predictions and measurements. In what follows, some background and discussion are provided to help with understanding the results. The details are found in the references and/or in the NASA final acoustic assessment report.

\section{III.A. Semi-Empirical Tools}

Some of the requirements desired for an aircraft noise prediction program include accuracy and speed of computations. Early in the design process, for example, this type of prediction program needs to be relatively easy to use and should yield a maximum amount of information from a minimum amount of input data and knowledge of the system. These conflicting criteria are a challenge to the capability and the accuracy of the program. The accuracy requirements of the program become more demanding as aircraft noise becomes more important. The ANOPP code has prediction models that are computationally efficient, yet of sufficient fidelity to capture the effects on noise of design and/or operational changes. To achieve this, ANOPP relies on semi-empirical methods for predicting the various aircraft noise sources. ANOPP provides the capability to (1) assess aircraft system noise, (2) assess aircraft component noise, and (3) evaluate aircraft noise technologies and flight procedures.

ANOPP consists of a set of functional modules that compute atmospheric properties, aircraft flight path, 
source-to-observer geometry, propagation of the source noise to ground observers, and community noise metrics, as well as predict the noise from the aircraft propulsion system and airframe. These modules are put together in a manner following the diagram shown in Figure 3. The modular design of ANOPP allows its prediction capabilities to be flexible and appropriate for problems ranging from isolated sources to complex flight regimes of varying conditions. In the examples of typical assessment results that follow, we will first show the isolated engine related source components of the fan and the jet. These will then be combined together with other components to predict the noise radiated from an engine system. Next, some typical results for the flap component of airframe noise will be presented and finally, the component noise sources will put together to predict the noise from a full aircraft. The most recent version (Level 25) of the Aircraft Noise Prediction Program (ANOPP) was used to assess the accuracy of the empirical methods for aircraft noise prediction.

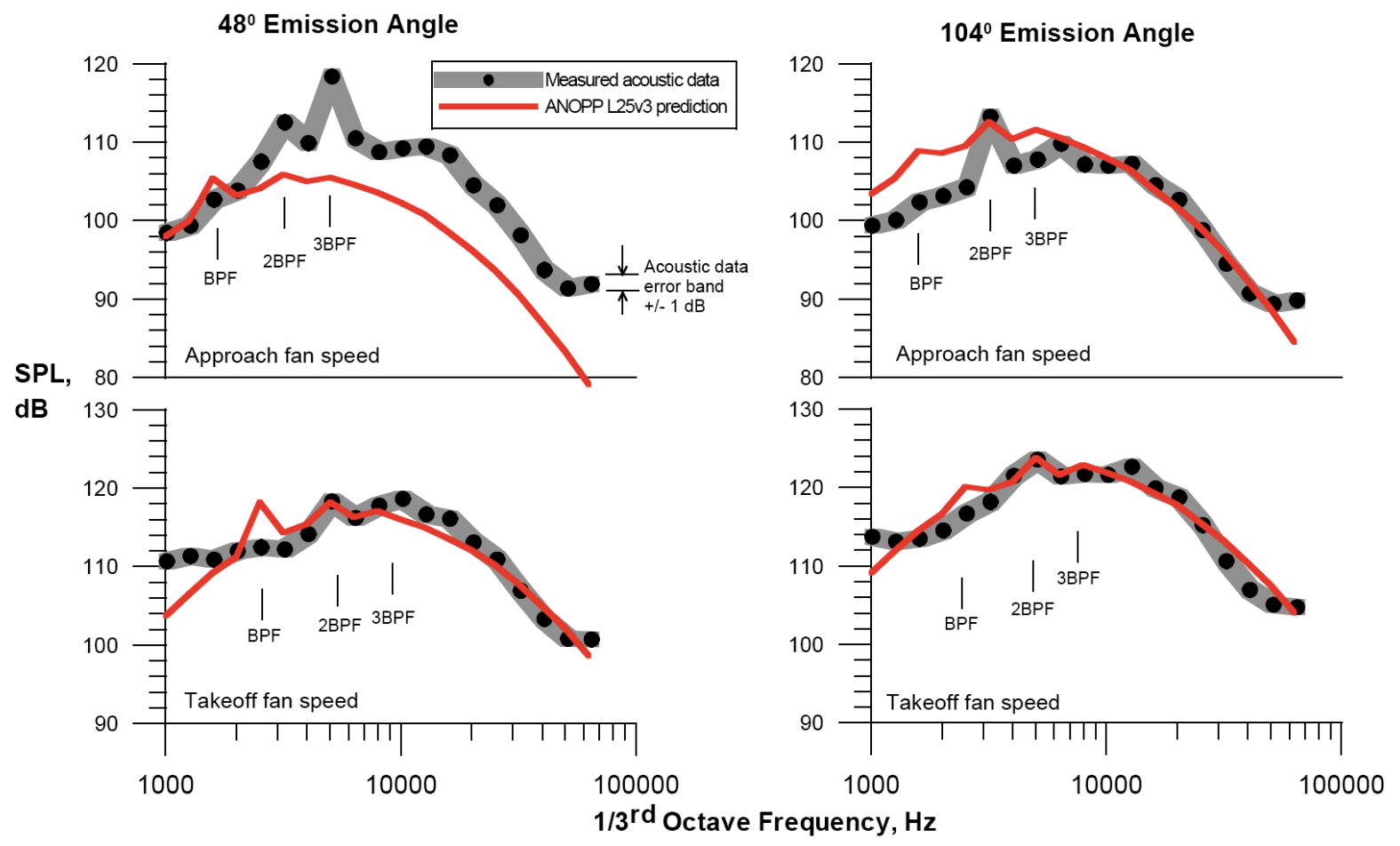

Figure 4. Comparison of measured and predicted 1/3-octave spectra for the ADP Fan 1 (1 $\mathrm{ft}$ radius, lossless).

\section{III.A.1. Fan Noise}

For fan noise prediction, the module in ANOPP, called HDNFAN, was used to predict the sideline noise radiated from the fans. The HDNFAN fan noise prediction module predicts both pure tones and broadband noise for the fan as functions of $1 / 3$-octave frequency bands and polar directivity angles using empirical relationships. The module has the option to use the original prediction method or extensions for small and large engines. ${ }^{2}$ The module accounts for six separate physical noise-generating mechanisms: inlet broadband noise, inlet rotor-stator interaction tones, inlet flow-distortion tones, combination tone noise, discharge broadband noise, and discharge rotor-stator interaction tones. This noise prediction module was constructed using a database of a number of production fans, but these databases did not include input from the model fan data used in this study.

The fan noise component predictions were compared to a database consisting of three selected 22-inch, model-scale fan test cases including a fan stage called the Advanced Ducted Propulsor (ADP) Fan 1 with a fan pressure ratio of 1.29, a second fan stage called the Source Diagnostic Test (SDT) fan with a fan pressure ratio of 1.47, and a third fan stage called the Quiet High Speed Fan 2 (QHSF2) with a fan pressure ratio of 1.8. All fans were tested in the NASA Glenn 9-Foot by 15-Foot low-speed acoustic wind tunnel. For the 
example here, results for sideline acoustic data with a hard-wall fan duct configuration will be shown for the ADP fan. The details may be found in Envia et al. ${ }^{16}$

Figure 4 compares measured 1/3-octave spectra for the ADP fan with the corresponding ANOPP fan noise module predictions at approach and take-off fan speeds at representative 48-deg and 104-deg sideline or emission angles from the fan inlet axis. It is common to show plots of this type, except for the inclusion of uncertainties, comparing predicted and measured spectra for a particular condition and at a particular directivity angle. The measured acoustic spectra were determined to have a maximum uncertainty of \pm 1 $\mathrm{dB}$. Thus, the acoustic data shown in Figure 4 shows this by drawing a $2 \mathrm{~dB}$ thick line to indicate this uncertainty in the measurements. A similar error analysis was performed for the ANOPP predictions by varying the aerodynamic input parameters by at least as much as suggested by an analysis of the accuracy of those parameters. The ANOPP noise predictions were found to have a less than $0.02 \mathrm{~dB}$ output variation for each of the input parameter variations. The comparison of the ANOPP prediction for ADP Fan 1 to experimental data at the higher fan speed is significant in that the Fan 1 cycle is outside of the fan database originally used to formulate ANOPP.

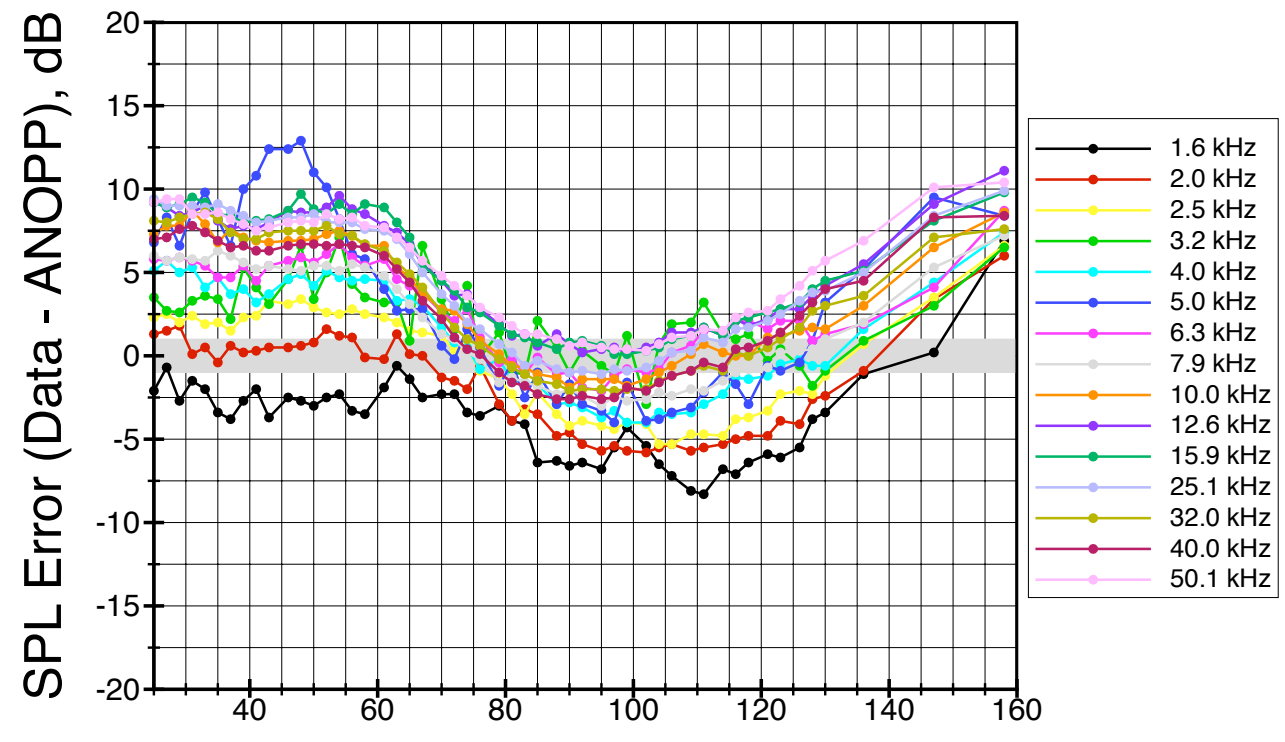

\section{Sideline Angle, deg.}

Figure 5. Comparison error between ANOPP predictions and measured data in dB for the ADP Fan 1 at approach conditions. Gray band represents $\pm 1 \mathrm{~dB}$ of measurement uncertainty.

To better illustrate the assessment of this fan noise prediction, a composite plot of the comparison error between ANOPP predictions and experimental data is shown in Figure 5 for the radiated ADP Fan 1 noise at approach conditions. In contrast to results shown in Figure 4, this plot attempts to convey the quality of the comparison at one operating condition by combining information about the amplitude error and uncertainty. The error is plotted for each 1/3-octave band frequency from 1.6 to $50.1 \mathrm{kHz}$ as a function of the sideline angle. The gray band represents the $\pm 1 \mathrm{~dB}$ of measurement uncertainty. Error levels greater than zero indicate that ANOPP under predicts the measured level and, conversely, ANOPP has over predicted the measured levels where the comparison error has a negative value. Clearly, the ANOPP fan module in this case most often under predicts the noise at most frequencies. However, we note that the error is consistent within about a $5 \mathrm{~dB}$ wide band for $1 / 3$-octave band frequencies above $3.2 \mathrm{kHz}$.

\section{III.A.2. Jet Noise}

The jet noise prediction empirical code assessed was the Stone Jet Noise Module (ST2JET) contained within the ANOPP aircraft noise prediction code. ${ }^{2}$ It is well documented, and represents the state of the art in acoustic prediction codes where virtual sources are associated with various aspects of jet flows and combined for a given case to predict the noise spectral directivity of a jet plume. These codes typically require input of 


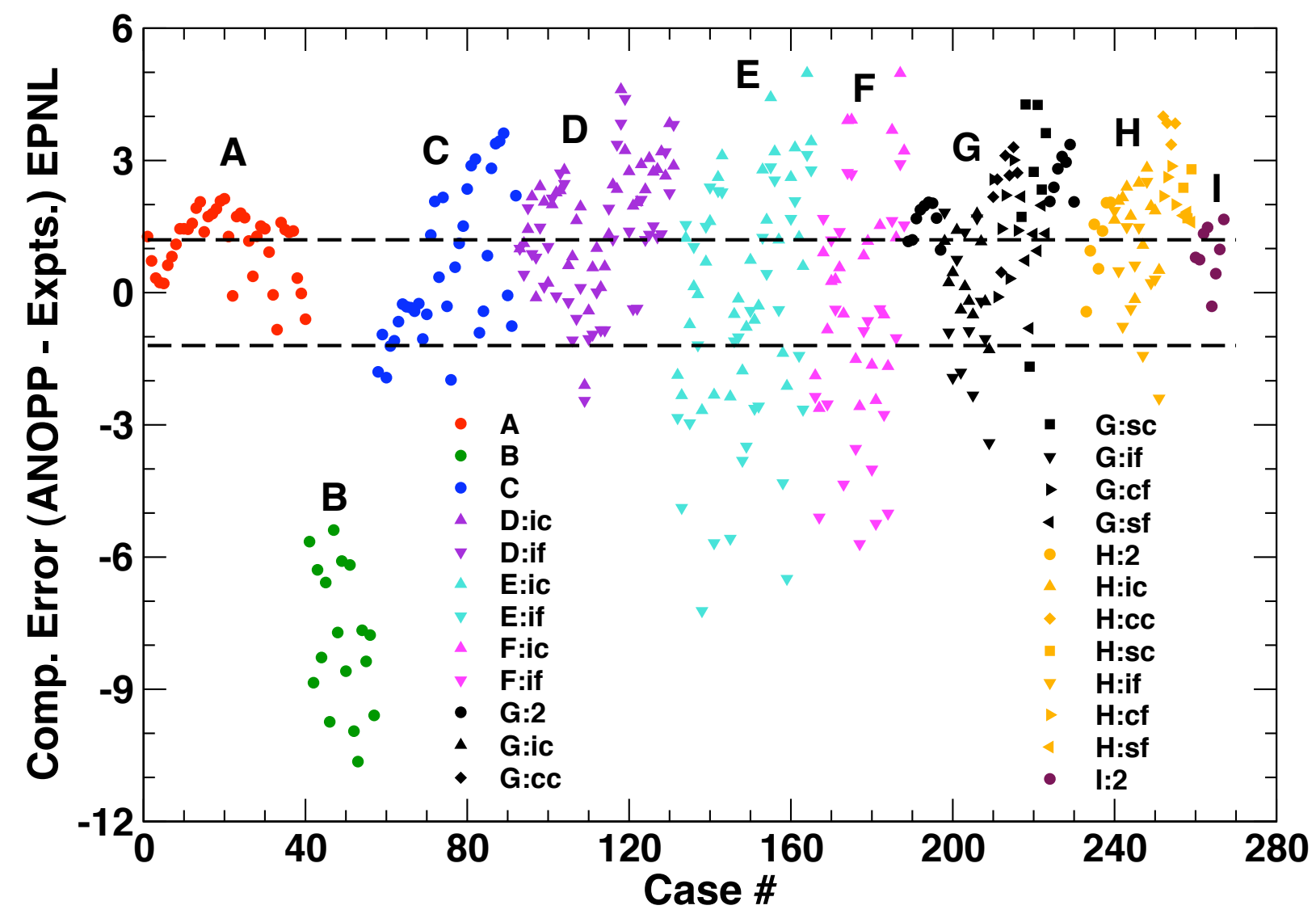

Figure 6. Comparison error between ANOPP predictions and measured data in EPNL for all jet noise cases in this assessment. Dashed lines represent $\mathbf{a} \pm 1.2$ EPNdB uncertainty band. Detailed legend in Table 2.

some description of the flow condition, such as velocity and temperature, and input of geometric parameters such as nozzle areas, extension lengths, and perhaps hydraulic perimeter in the case of enhanced mixing devices. A total of 265 jet noise cases were run on the ST2JET code, some with internal mixers and the remainder without mixers. The internally mixed nozzle systems were approximated two different ways by the code, leading to a total of 407 analyzed jet noise cases.

ST2JET is based on the semi-empirical model developed by Stone et al. ${ }^{17}$ The method was developed from data with bypass ratios ranging from 5.0 to 14.9 obtained from free-jet facilities at NASA Glenn Research Center and NASA Langley Research Center as well as the anechoic free-jet facilities at Lockheed and General Electric. ST2JET is an update to the older STNJET module in ANOPP and extends the ANOPP jet noise prediction capability to higher bypass ratio engines for a full range of nozzle configurations including jets with inverted velocity profiles. The prediction method combines the component noise sources into a single one-third octave band spectrum at each directivity angle.

A significant measure of jet noise is the Effective Perceived Noise Level (EPNL). This metric is tied to the scale of the jet and gives more weight to the frequency bands near $2000 \mathrm{~Hz}$. These frequencies most likely are different from the typical peak frequency of jet noise. This penalizes codes which predict the peak jet noise level well but do not predict the noise levels at frequencies away from the peak that contribute to the important perceived noise from large aircraft. When the EPNL is computed, it combines the effects of amplitude, frequency, directivity, duration, and perceived noisiness of the source. It integrates these effects into a single number that may be used to compare many different cases. Consequently, the individual details are lost. To present the error in EPNL, all cases were transformed to a 1 meter diameter nozzle in level flight at Mach number 0.28 and an altitude of 1640 feet. Using this metric, a summary of the comparison error result for the 265 jet noise cases is shown in Figure 6. More details may be found in Bridges et al. ${ }^{18}$

In this figure, the difference between the ANOPP predictions and the experimental data in terms of EPNL are plotted as a function of the case number. A listing of some details regarding the labels in the figure is 


\begin{tabular}{|c|c|c|c|c|c|c|}
\hline Label & Exit Geometry & Axial Geometry & BPR & Ref. M & \# of Expts. & Modeling Approx. \\
\hline A & Axisymmetric & Single Stream & 0 & $<1$ & 40 & \\
B & Chevrons & Single Stream & 0 & $<1$ & 17 & \\
C & Axisymmetric & Single Stream & 0 & $>1$ & 35 & \\
D:ic & Axisymmetric & Internal Splitter & 0.1 & $\lessgtr 1$ & 39 & Coplanar Approx. \\
D:if & Axisymmetric & Internal Splitter & 0.1 & $\lessgtr 1$ & $(39)$ & Fully Mixed Approx. \\
E:ic & Axisymmetric & Internal Splitter & 1 & $\lessgtr 1$ & 34 & Coplanar Approx. \\
E:if & Axisymmetric & Internal Splitter & 1 & $\lessgtr 1$ & $(34)$ & Fully Mixed Approx. \\
F:ic & Axisymmetric & Internal Splitter & 2 & $\lessgtr 1$ & 23 & Coplanar Approx. \\
F:if & Axisymmetric & Internal Splitter & 2 & $\lessgtr 1$ & $(23)$ & Fully Mixed Approx. \\
G:2 & Axisymmetric & Separate Streams & 5 & $<1$ & 16 & \\
G:ic & Axisymmetric & Internal Splitter & 5 & $<1$ & 12 & Coplanar Approx. \\
G:cc & Axisymmetric & Internal Chevrons & 5 & $<1$ & 7 & Coplanar Approx. \\
G:sc & Axisymmetric & Int. Scalloped Lobes & 5 & $<1$ & 7 & Coplanar Approx. \\
G:if & Axisymmetric & Internal Splitter & 5 & $<1$ & $(12)$ & Fully Mixed Approx. \\
G:cf & Axisymmetric & Internal Chevrons & 5 & $<1$ & $(7)$ & Fully Mixed Approx. \\
G:sf & Axisymmetric & Int. Scalloped Lobes & 5 & $<1$ & $(7)$ & Fully Mixed Approx. \\
H:2 & Axisymmetric & Separate Streams & 8 & $<1$ & 7 & \\
H:ic & Axisymmetric & Internal Splitter & 8 & $<1$ & 12 & Coplanar Approx. \\
H:cc & Axisymmetric & Internal Chevrons & 8 & $<1$ & 4 & Coplanar Approx. \\
H:sc & Axisymmetric & Int. Scalloped Lobes & 8 & $<1$ & 4 & Coplanar Approx. \\
H:if & Axisymmetric & Internal Splitter & 8 & $<1$ & $(12)$ & Fully Mixed Approx. \\
H:cf & Axisymmetric & Internal Chevrons & 8 & $<1$ & $(4)$ & Fully Mixed Approx. \\
H:sf & Axisymmetric & Int. Scalloped Lobes & 8 & $<1$ & $(4)$ & Fully Mixed Approx. \\
I:2 & Axisymmetric & Separate Streams & 11 & $<1$ & 8 & \\
\hline
\end{tabular}

$2=$ Separate Streams, ix = Internal Splitter, $\mathrm{cx}=$ Internal Chevrons, $\mathrm{sx}=$ Internal Scalloped Lobes, $\mathrm{xc}=$ Coplanar Approximation, $\mathrm{xf}=$ Fully Mixed Approximation,

$(\mathrm{n})=$ same expt. data as coplanar approximation

Table 2. Definition of labels used for the jet noise cases shown in Figure 6.

given in Table 2. As mentioned earlier, the prediction code can model internally mixed nozzle systems in two different ways. The table lists those ways as the coplanar approximation and the fully mixed approximation. Both approximations were used for the jet case families labeled $\mathrm{D}$ to $\mathrm{H}$ where the nozzle internal mixer was either a splitter, chevrons, or scalloped lobes. Figure 6 also shows a \pm 1.2 EPNdB uncertainty band, lying between the dashed lines, that represents an estimated value based on the uncertainty in a measured jet noise spectrum at a frequency, with the proper scaling, that dominates in the EPNL calculation. In general, for an axisymmetric type nozzle that does not contain chevrons or scalloped lobes, the EPNL predictions agree with the measurements within the level of uncertainty about $40 \%$ of the time for these cases. The predictions for the nozzles with those mixers drop to $10 \%$ agreement with the measurements.

These sample results in terms of EPNL values are often off by several EPNdB. However, for axisymmetric jets, the ANOPP code gave similar and fairly low levels of error for a broad range of axisymmetric jet flows. The ST2JET module was applicable to a wide range of flow conditions, predicting overall sound pressure levels only slightly outside of experimental uncertainty, within $2 \mathrm{EPNdB}$, for most axisymmetric jets. This includes jets with both single streams and two separate flow streams. Supersonic jets and jets with mixers had much larger comparison errors using this EPNL metric. 


\section{III.A.3. Engine System Noise}

Figure 3 may be used to illustrate a typical ANOPP run for a static-engine test where only the engine source noise modules are used. The first step defines the ambient conditions such as pressure, temperature, relative humidity, and related properties like the speed of sound. These properties are varied with altitude in an atmospheric module and also used to determine the amount of sound attenuation by atmospheric absorption.

The second step uses the engine noise-source modules to predict the noise as functions of $1 / 3$-octave bands and polar directivity angles. For a static-engine test, the modules are: ${ }^{2}$

1. HDNFAN - This module predicts both broadband noise and the contribution of tones with a choice of empirical methods.

2. TREAT - This module updates the fan-noise prediction by accounting for acoustic treatment. The module has a choice of treatment methods.

3. GECOR - The combustion-noise module predicts broadband noise with a choice of empirical methods. ${ }^{19,20}$

4. TUR - The turbine-noise module predicts both broadband and tone noise using empirical relationships. $^{20,21}$

5. STNJET - This noise-source module predicts jet mixing noise and shock associated noise for circular jets. It was used to predict the noise from a subsonic single stream jet nozzle with no center plug.

The final step propagates the noise from the source location to the actual observer location. The propagation stage is assumed to be linear. A propagation module sums the noise data that are generated by the noise-source modules and transfers them to the observer positions. For a static-engine test, this includes accounting for spherical spreading and atmospheric attenuation. In a typical static-engine test, the engine is mounted on a stand a certain distance above a hard-surface ground and the acoustic-measurement microphones are ground mounted to minimize, if not fully remove, the ground reflection effect.

The sensitivity to uncertainty in the performance parameters for an ANOPP simulation is determined as follows: For a specific engine operation point, approach, cutback, or take-off, each performance parameter is independently varied up and down a small amount to determine the sensitivity to uncertainty in that parameter. The absolute values of the individual sensitivities are then added together to yield an upper bound on the prediction sensitivity relative to a $1 \%$ or a $3 \%$ uncertainty in the performance parameters.

The NASA funded Engine Validation of Noise and Emission Reduction Technology (EVNERT) program $^{22,23}$ was completed, in part, to gather an extensive set of acoustic far-field data to characterize engine noise sources, to gain insight into their mechanisms, and to evaluate select noise reduction concepts. The tests were carried out by Honeywell Aerospace at their San Tan outdoor engine test facility. The Honeywell TECH977 research engine, which is characteristic of small engines in the 6,000-8,000 lbs thrust class, was used in these tests.

Data comparisons between a hard-wall configuration and a treated-wall configuration are shown in Figures 7 and 8 for approach and take-off conditions, respectively. The hard-wall configuration, shown in the left column, was a baseline case where the fan flow path had all hard-wall conditions. The treated-wall configuration, shown in the right side, was identical except single-degree-of-freedom acoustic liners were installed in the inlet, aft-fan c-duct, and nozzle.

As was done for the fan noise comparisons in Figure 4, 1/3-octave, sound pressure level, SPL, spectral comparisons are made here for a single operating condition and at a particular directivity angle. Figure 7 shows spectra at the approach speed (60\% corrected fan speed). Even though the four engine source prediction modules listed above were used, the predicted results are dominated by fan and jet noise. Hence, the three directivity angles in the figure represent changes from forward of the engine (30 degrees), to sideline, and to aft of the engine (150 degrees). The dominance of fan noise forward and jet noise aft can be seen in the results. How speed effects these spectral results is seen by comparing Figure 7 to Figure 8 where the results are at the take-off speed ( $87 \%$ corrected fan speed). The predictions show many similarities with the measured data, from the low frequency peak at the 150-deg angle due to jet noise to the decrease in high frequency noise levels at most angles when acoustic treatment is present. In the plots, the ANOPP predictions have uncertainty bands $(1 \%$ and $3 \%$ of performance parameter uncertainty, dark and light gray, respectively) and the static-engine-test data have error bars based on estimated calibration/instrumentation 
Hard Wall
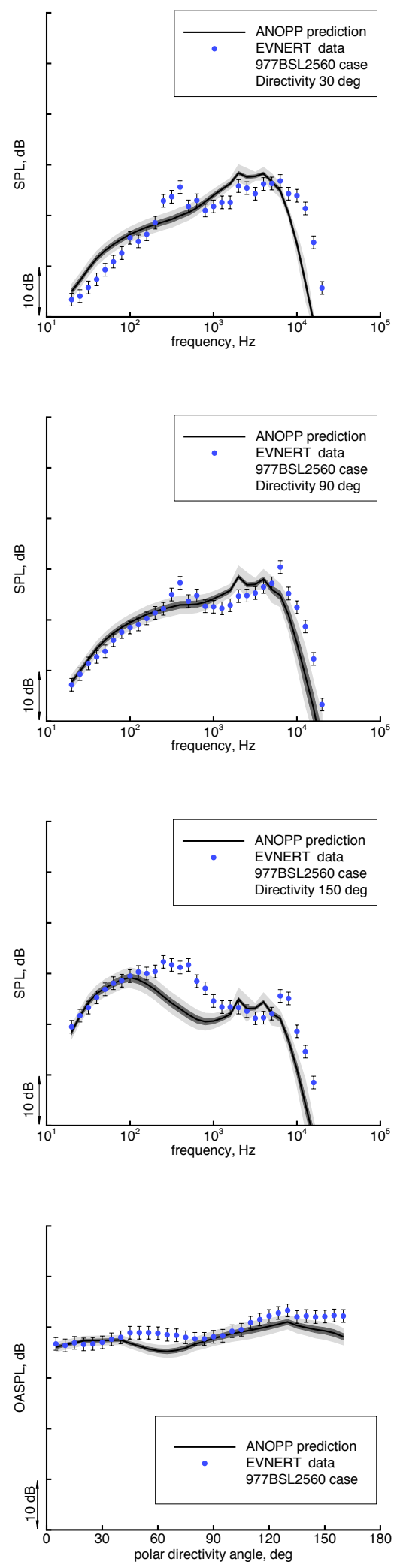

\section{Treated Wall}
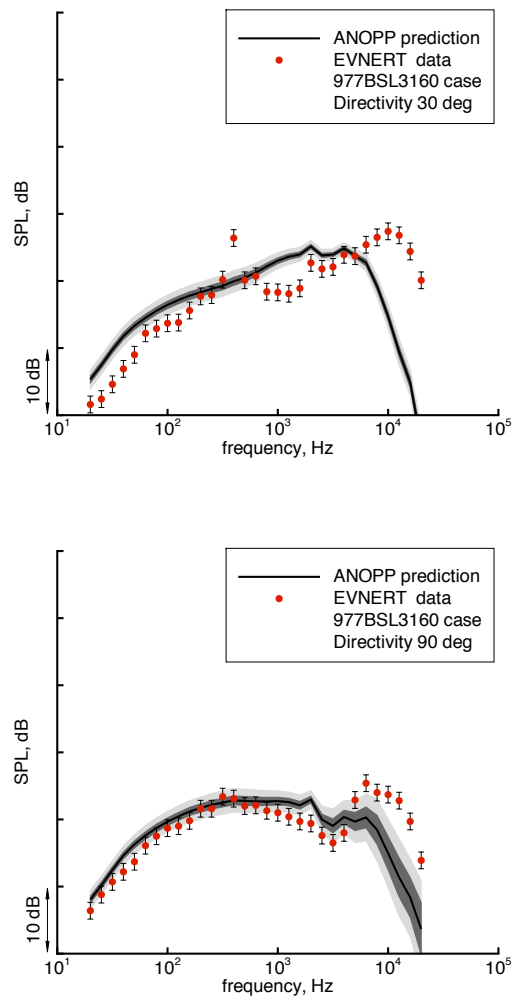

90 Deg

Spectra

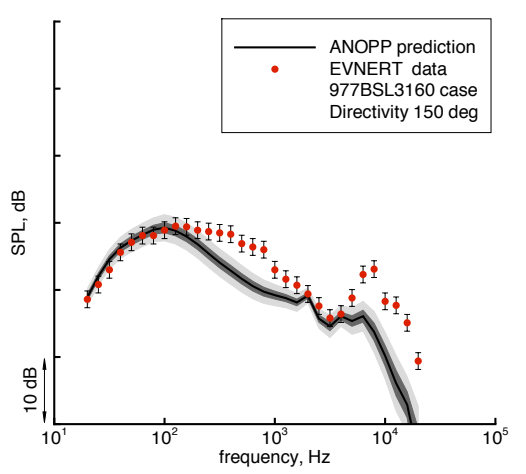

150 Deg Spectra

\section{Deg \\ Spectra}
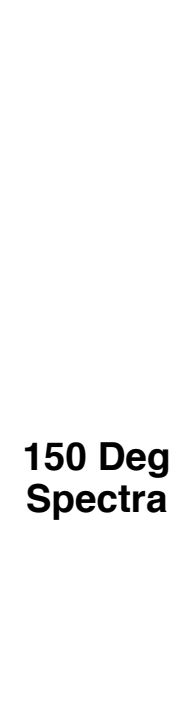

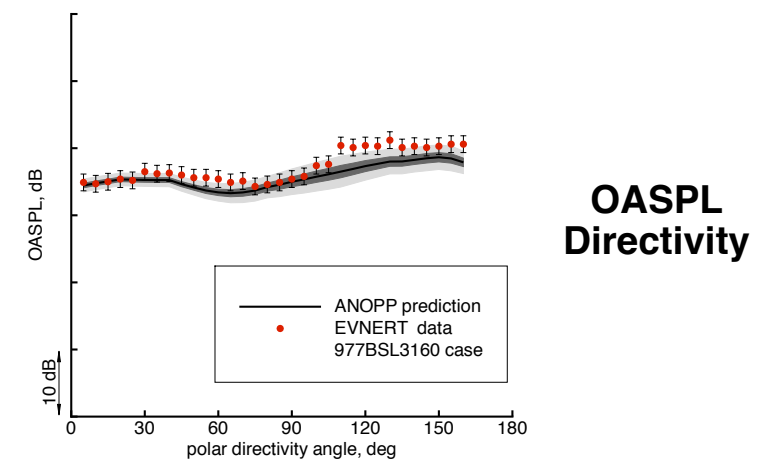

Figure 7. 1/3-octave spectra at three directivity angles and OASPL directivity comparisons between measured data and ANOPP predictions with two different duct wall treatments. Approach conditions at $60 \%$ corrected fan speed. Prediction uncertainty bands: $1 \%$ input uncertainties (dark gray), 3\% input uncertainties (light gray). 
Hard Wall
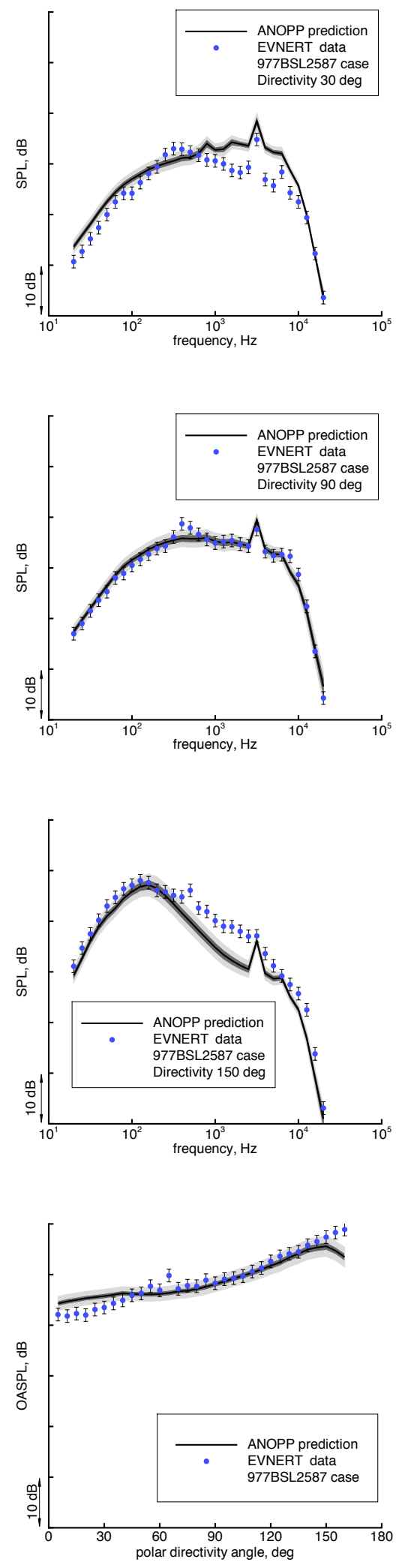

\section{Treated Wall}
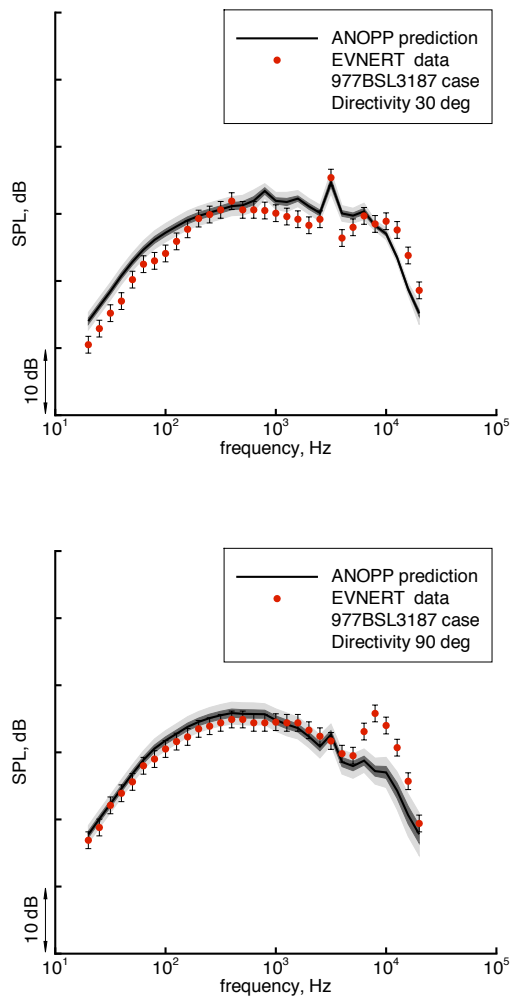

90 Deg

Spectra

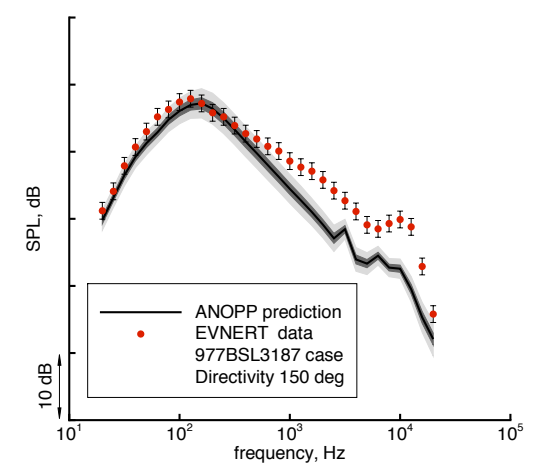

150 Deg Spectra

\section{Spectra}
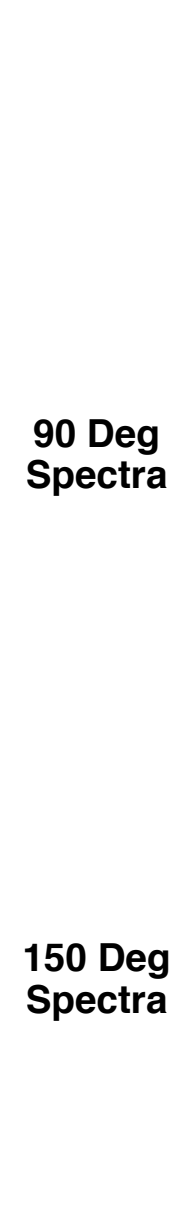

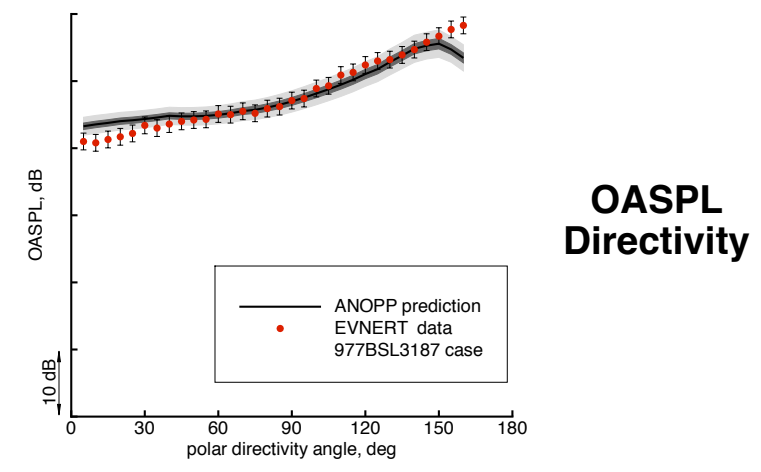

Figure 8. 1/3-octave spectra at three directivity angles and OASPL directivity comparisons between measured data and ANOPP predictions with two different duct wall treatments. Take-off conditions at $87 \%$ corrected fan speed. Prediction uncertainty bands: $1 \%$ input uncertainties (dark gray), 3\% input uncertainties (light gray). 
uncertainty. Considering the semi-empirical bases of the ANOPP predictions, the spectral comparisons show similar shapes and show agreement within uncertainty when a particular source dominates the noise. However, there are plenty of examples where the predictions differ from measured values at particular frequencies by as much as 10 decibels. Finally to illustrate what can happen for an integrated result, these figures show the overall sound pressure levels (OASPL) as a function of the directivity angle. It can be seen that whenever the spectral prediction captures the bulk of the peak energy, then the predicted OASPL often compares well with the measured value within the margin of uncertainty.

\section{III.A.4. Airframe Noise}

The noise radiated from an aircraft is most influenced by airframe sources during airport approach, when its engines are operating at reduced thrust, and the airframe itself is configured for landing. During landing operations, the aircraft's wings are in a high-lift configuration, and its landing gear is lowered. Various model and full-scale tests have revealed that the prominent sources of airframe noise are associated with the leading-edge slats, trailing-edge flaps, and landing gear of commercial transports.

The assessment study of NASA's current capabilities in airframe noise prediction included comparisons between predictions and measured data for flap, slat and landing gear related noise sources. Far-field acoustic radiation from these sources was calculated for model-scale airframe components as well as fullscale operational aircraft. These predictions were compared with acoustic measurements from various test programs. The examples presented here illustrate a comparison between a code assessment using model-scale test data and a code assessment using full-scale flight test data. At model scale, the data were from a wind tunnel test conducted of an airfoil section with a partial-span flap. ${ }^{24}$ Full-scale flap data came from a flight test using a Gulfstream G550 at the NASA Wallops Flight Facility. ${ }^{25}$

Airframe noise modules are available in ANOPP, each containing several component source models that can be applied in isolation or appropriately summed to compute the total non-propulsive noise for a given aircraft configuration. An example of these airframe noise modules contains acoustic source models developed by Fink. ${ }^{26,27}$ In this noise component method, total airframe noise is calculated as an acoustic sum of component sources. It is assumed that all component sources are mutually incoherent. The noise contribution for each airframe component was developed with an appropriate scaling law and the amplitudes were determined by matching with available experimental data. The ANOPP documentation ${ }^{2}$ contains further details on this module.

The 1/3-octave band flap-edge noise spectra, used for comparison to ANOPP predictions, were obtained from measurements of a model-scale airfoil with an attached flap in the NASA Quiet Flow Facility (QFF) ${ }^{24}$ The flap angle was set to either 29 degrees or 39 degrees during the test. The effects of Doppler frequency shifting were removed from the ANOPP output for comparison to the wind tunnel data. Using the Fink

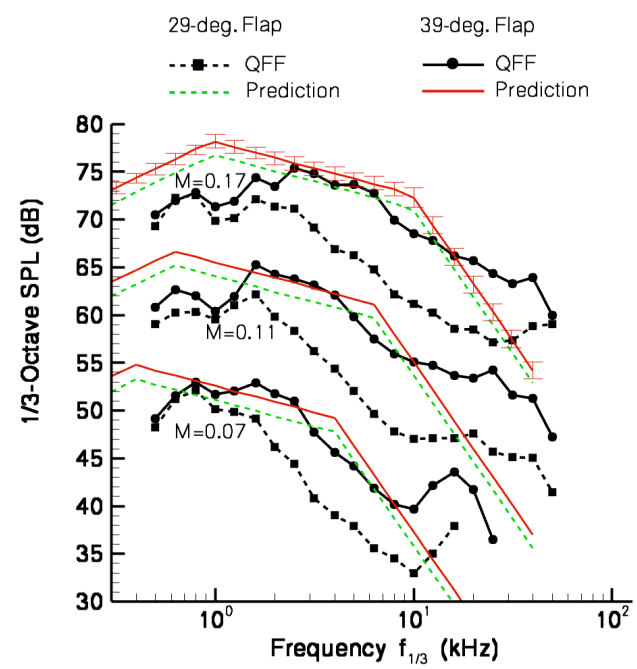

Figure 9. Comparison between flap noise measurement and prediction using the Fink method.

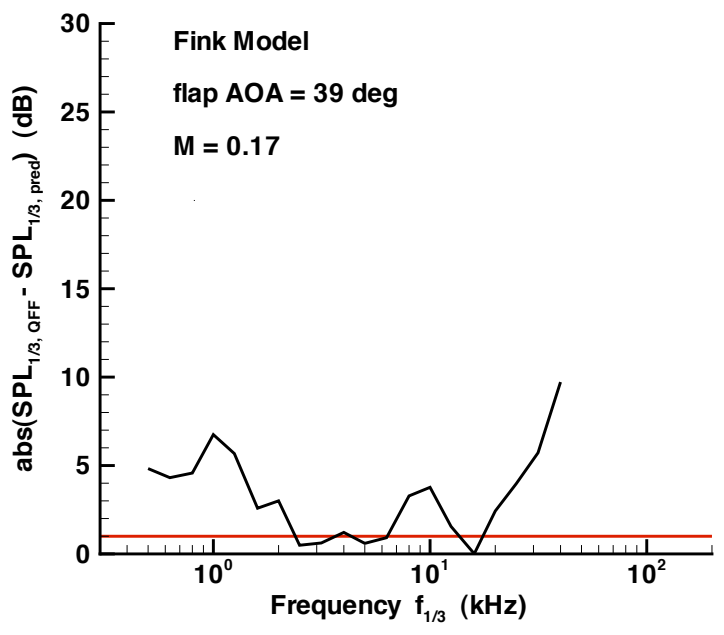

Figure 10. Comparison error between prediction and measurement for results in Figure 9. Red line represents extent of $1 \mathrm{~dB}$ uncertainty band on the absolute scale. 
model for flap noise, ANOPP generated the predicted spectra which are compared to the QFF spectra in Figure 9. It is seen that the predicted sound pressure levels are closer to the measured levels over a broad frequency range for the 39-deg flap, while the levels are over predicted for most of the frequency range for the 29-deg flap case. The uncertainty was estimated for both the predicted and the measured values. For the predictions, a sensitivity study was performed over a limited set of parameters for the case where the flap angle was set at 39 degrees and the Mach number was 0.17 . The Mach number was varied $\pm 1 \%$ and the wing angle of attack was varied $\pm 5 \%$. The effect from these parameter variations on the spectral levels is shown by the error bars in Figure 9. A series of 16 data sets containing flap noise spectral results were used to determine the uncertainty of the measurements. The results of the analysis for the same conditions as in the predictions indicated that the maximum measured sound pressure level standard deviation was $\pm 0.19 \mathrm{~dB}$, a value that is within the size of the measurement symbols in Figure 9. The absolute value of the comparison error between the experimental and predicted spectra is plotted in Figure 10 for the 39-deg flap and a Mach number of 0.17 . This figure also shows the extent of the estimated $1 \mathrm{~dB}$ uncertainty band due primarily to the uncertainty in the predictions. For this case, which represents an aircraft flap during approach conditions at model scale, the error is small or within uncertainty between $2.5 \mathrm{kHz}$ to $6 \mathrm{kHz}$. However, we note that ANOPP was unable to predict the change in noise due to the change in flap deflection angle.

To illustrate the changes that occur when using the semi-empirical method to predict a full-scale flight test, we use the data collected from the recent flight test program jointly conducted by NASA and Gulfstream at NASA's Wallops Flight Facility. Acoustic data for a Gulfstream G550 aircraft on approach to landing were acquired using both a ground-based microphone array and individual microphones. At least twenty-four varying combinations of aircraft configurations and test conditions were flown. The test matrix, ranging from cruise to landing configurations, was designed to provide an acoustic characterization of both the full aircraft and individual airframe components. Noise sources were isolated by selectively deploying components (flaps, main landing gear, nose gear, spoilers, etc.) and altering the airspeed, glide path, and engine settings. Details may be found in Khorrami et al. ${ }^{25}$

For each configuration, noise measurements were obtained at three distinct speeds with multiple runs at each speed in order to ensure that statistically meaningful data were obtained. Given the large number of conditions tested, only select configurations at the nominal speed of 147 knots and only measurements for the aircraft directly overhead were used to perform the assessment. For the purposes of this illustration, only the configurations with the flaps deployed at a 39-deg deflection angle and the landing gear up (labeled Flaps 39, LG UP) and the flaps retracted and the landing gear up (clean configuration) are considered.

The Fink model in ANOPP was also utilized to make the full-scale airframe noise predictions. In contrast to the acoustic measurements in ground based facilities, in flight, the aircraft represents a moving noise source. To partially simulate the moving source behavior, several other ANOPP modules were utilized, in addition to the airframe noise module. Appearing in the sequence used in the prediction process, they were: the Atmospheric, Atmospheric Absorption, Steady Flyover, Geometry, Airframe Noise, and Propagation modules. The predictions were performed for a standard day (70 F and $70 \%$ relative humidity) conditions, reference altitude of 394 feet, aircraft speed of $147 \mathrm{knots}(248 \mathrm{ft} / \mathrm{sec}$ ), azimuth (sideline or phi) angle of 0 degree, and polar (overhead) angle of 90 degrees. For the sample results presented here, the full-scale noise assessment of a regional size jet is shown in terms of relative noise levels. The sound pressure levels for the clean configuration are subtracted from the sound pressure levels associated with the flaps-deployed configuration. This relative noise metric partially eliminates any ambiguity attributed to the presence of residual engine noise or the background environmental noise within the measured signal.

The sound pressure levels for the two selected conditions are plotted in Figure 11. Each curve represents the averaged value of all the runs conducted for that particular configuration. Comparison of the levels between the flaps-deployed and the clean configurations shows the noise contribution from the deflected flaps over most of the frequency range. Given that all the flights were executed with an approach-idle engine setting, Figure 11 indicates that residual engine noise was not a factor.

The predicted 1/3-octave sound pressure level spectra for the two selected configurations are shown in Figure 12. In the low to mid frequency range, the computed spectra are similar in shape to the measured values. In particular, the frequencies corresponding to the peak level are well predicted. For frequencies above $800 \mathrm{~Hz}$, however, the predicted spectra begin to drop in level, with the decay becoming most rapid above $2 \mathrm{kHz}$.

An assessment of the ability to predict the full-scale airframe noise with just the flaps deployed is shown by the comparison error in Figure 13. The error is computed from the difference between the relative 


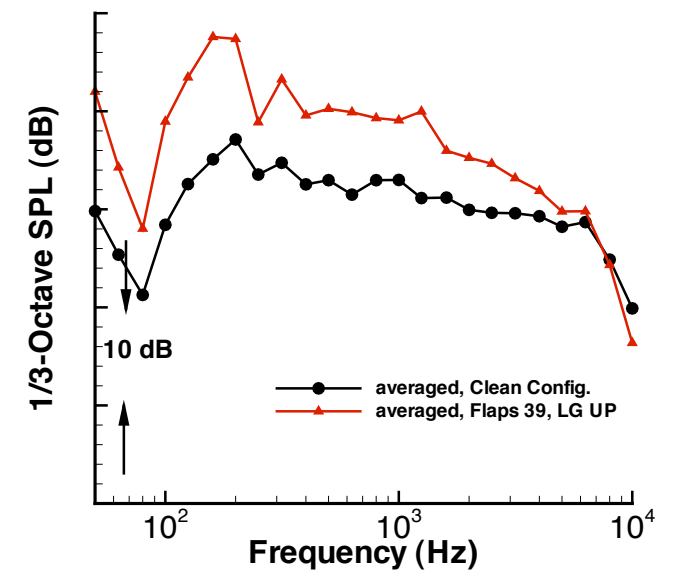

Figure 11. Averaged measured spectra for the flapsdeployed airframe configuration and the clean configuration.

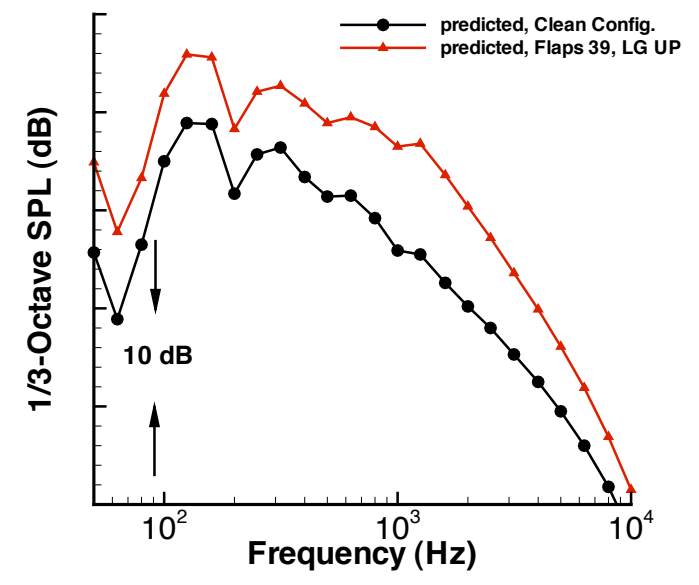

Figure 12. Predicted spectra for the flaps-deployed airframe configuration and the clean configuration.

measured spectra and the relative predicted spectra. The gray area represents an estimated uncertainty band of $\pm 2 \mathrm{~dB}$ due primarily to measurement uncertainty. In this figure, positive values indicate under prediction and negative values over prediction of the measured sound pressures. The plot in Figure 13 shows that at frequencies below $3 \mathrm{kHz}$, the error in relative level is mostly within plus or minus 5 dB. The errors in the relative noise levels at high frequencies (above $3 \mathrm{kHz}$ ) must be viewed with caution due to the presence of high background noise levels and the absence of atmospheric corrections in the measured pressures. There is some similarity between this result and the model-scale result shown in Figure 10. A large error at low frequency is followed by a mid-frequency region with relatively low error and then the error increases with higher frequency.

\section{III.A.5. Aircraft System Noise}

The prediction of aircraft noise by the ANOPP system is summarized by the four steps indicated in Figure 3. These steps are used to determine the noise of an aircraft that follows an arbitrary fight path in the presence of an observer on the ground. The first step within the ANOPP process is to provide the Aircraft Flight Definition. The Atmospheric Module establishes the properties of the atmosphere (pressure, density, and humidity as functions of altitude). The Flight Dynamics Modules create a flight path, or one is externally provided, wherein the time-dependent power setting is used as the control function. The Geometry Module then computes the time dependent emission angles and source observer distances. The flight path definition also defines the engine state at each point along the flight path.

Once the aircraft flight path and condition are defined, the second step is to compute the noise from each

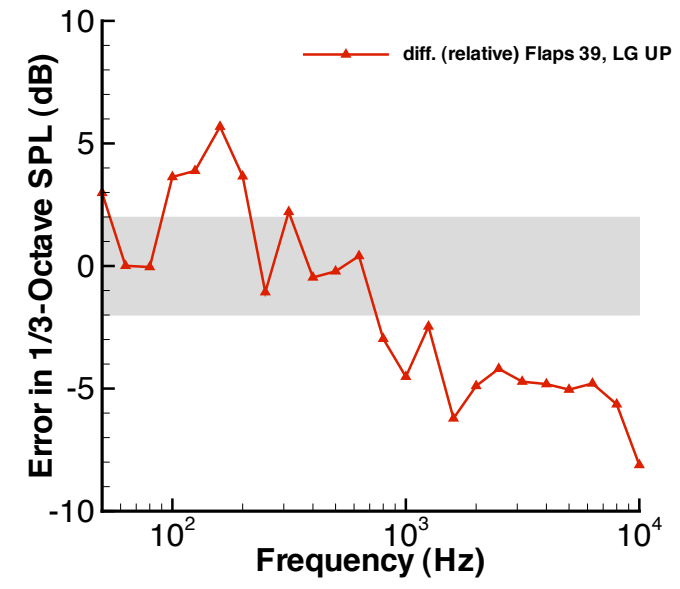

Figure 13. Comparison error between measured and predicted relative spectra. Gray area represents an estimated $\pm 2 \mathrm{~dB}$ uncertainty band. propulsion and airframe source. Each noise source is given in a specified axis system so that each observer vector will be expressed in several source coordinate systems at the same time. Source noise predictions are made in several coordinate systems which are attached to the aircraft. The ANOPP executive and source noise modules then convert to a common coordinate system to propagate and properly compute the (total) noise at specified observer locations in the third step. The system internally handles the conversion between the source noise coordinate systems and the observer coordinate system. Using an assumption of acoustic superposition, the sources are analytically 
added together in the vicinity of the aircraft and propagated to observers on the ground. ANOPP computes $1 / 3$-octave band sound pressure level spectra from all major aircraft noise sources for both static and in-flight conditions. Finally in the last step, all the computed levels may be rolled up into a variety of higher-order noise metrics such as the Effective Perceived Noise Level (EPNL).

The sample result illustrates the difficulty of doing an assessment of the capability to predict the noise radiated from a complete aircraft. The lack of available data with precise performance and acoustic measurements degrades the quality of the results. Nonetheless, there is certification noise level data that can be used for assessment purposes. Here, aircraft noise predictions from the ANOPP code are compared to measured certification data for a two-engined aircraft following regulated procedures.

The aircraft is flown through standardized take-off

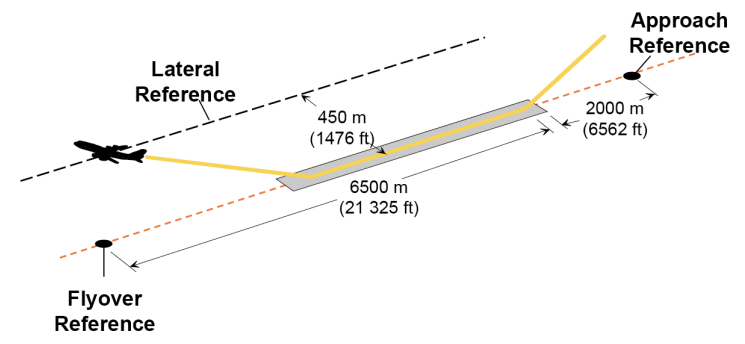

Figure 14. Aircraft flight path for noise certification measurements and location of measurement points. and approach procedures and the $1 / 3$-octave band fre-

quency spectrum time histories are measured at three locations identified as lateral sideline, flyover, and approach. These locations are shown on the flight path diagram in Figure 14. On take-off, the aircraft must climb at full power and take-off flaps to an altitude of at least 984 feet (for a two engine aircraft), at which point it may execute a noise abating cutback maneuver as long as the thrust is sufficient to maintain a $4 \%$ climb gradient or engine-out level climb. On approach, the aircraft must fly along a three degree descent slope at constant speed with gear and flaps down. For both take-off and approach procedures, the aircraft must be at its maximum weight for which it is to be certified.

\begin{tabular}{|l|c|c|c|}
\hline & ANOPP & Cert. Data & Comp. Error \\
\hline Approach EPNL (EPNdB) & $95.6 \pm 1.3$ & 96.5 & -0.9 \\
Flyover EPNL (EPNdB) & $90.8 \pm 0.2$ & 86.8 & 4.0 \\
Lateral EPNL (EPNdB) & $92.3 \pm 0.4$ & 93.6 & -1.3 \\
\hline
\end{tabular}

Table 3. Predicted EPNL values with uncertainty range compared to certification point data.

ANOPP aircraft noise predictions for the three certification points are shown in Table 3 compared to measured certification data for a Boeing 737 aircraft equipped with CFM56-7B type engines. For the predictions, the Boeing 737 had a take-off gross weight of 174,200 pounds and a landing weight of 146,300 pounds. The predictions were based on 21 simulations with varying input parameters resulting in the $95 \%$ confidence interval of uncertainty listed in the table. For the most part, the predicted noise at all three certification points is dominated by the engine noise sources, i.e. fan noise and/or jet noise. The only other significant source is flap noise on approach. Since much of the performance data on the CFM56 engine is proprietary, the propulsion system performance data that is required as ANOPP input was obtained from a propulsion system simulation program. This could easily result in performance values that are dissimilar to actual engines and be a part of why there are discrepancies between measured and predicted noise levels.

To further illustrate the difficulty in assessing the quality of the aircraft noise predictions, consider that fan noise, jet noise, and flap noise were listed in the example as dominant noise sources. For each of these components, the comparison of ANOPP predictions with measured data were shown previously to have potentially larger errors and uncertainties than what is shown for the aircraft assessment. Part of the reason for discrepancy may be explained using the engine system example where it was shown that the OASPL could be predicted as long as the peak noise of the source spectrum was captured regardless of the detailed spectral comparisons.

\section{III.B. Analytical Tools}

Analytical methods rely on the fundamental fluid equations and on the modeling of physical phenomena to simulate the noise generation and propagation processes. Mathematically, the exact equations of the motion are rearranged so that a linear wave equation is obtained whose left hand side describes the propagation of sound and its right hand side represents a known aerodynamic source that generates the sound, thus 
separating the acoustics from the aerodynamic source of the problem (i.e. an acoustic analogy). The aerodynamic source is to be measured, computed or otherwise modeled independently. The solution to the wave equation is given formally in terms of integrals that describe convolution of the source distribution and propagation characteristics. Depending on the level of approximations involved in the description of the fan geometry and/or flow conditions, the solution can be expressed either in closed form, or may require the use of quadrature schemes to evaluate the solution integrals. The overall fidelity of these models depends on the level of approximations used to describe the sources, the fan geometry and flow conditions. Such models have been developed for a number of fan noise sources, but most have been developed to predict the rotor-stator interaction noise, which is used to predict tone or broadband noise produced as a result of the interaction between the fan wakes and fan exit guide vanes. Here, the most recent version of the RSI (Rotor-Stator Interaction) code was chosen to predict broadband noise generated by the interaction of rotor turbulence impinging on the downstream stator (in the bypass duct). The underlying theory for this code has been described in detail in Nallasamy and Envia. ${ }^{7}$

The RSI code requires a description of the impinging turbulence characteristics (i.e., intensity, length scales, etc.) as input for computing noise spectra. Such information is typically available from experiments or is heuristically modeled to provide the best fit of the predicted noise spectra to the measured levels. An alternative approach, used here, was to compute the turbulence characteristics using a RANS CFD calculation, since measured turbulence data are often difficult to obtain. The CFD solution was used to construct a description of the root-mean-squared turbulence intensity across the pitch, to determine the integral length scales of turbulence, to circumferentially average the mean axial velocity, and to circumferentially average the mean tangential velocity at a number of radial stations spanning the vane. Together with some basic geometric information about the vane geometry, these constituted the input necessary to run the RSI code. ${ }^{7}$

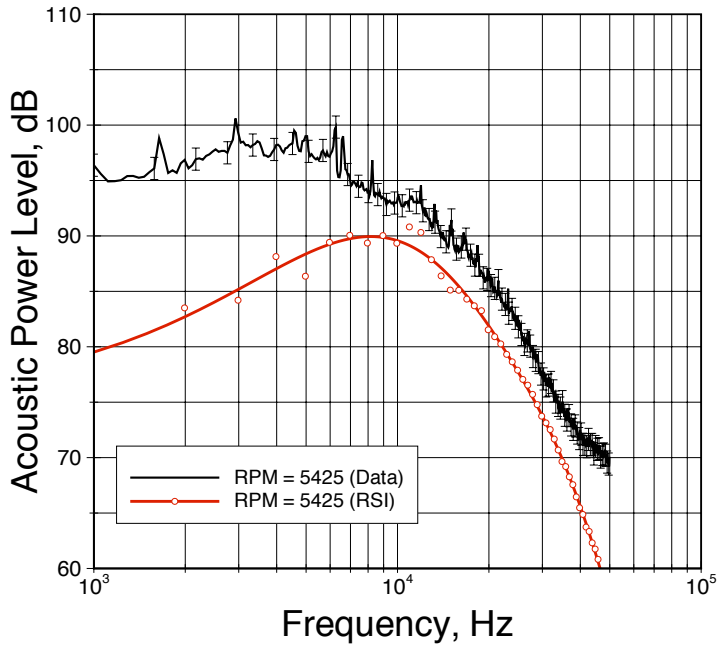

Figure 15. Narrow band acoustic power level comparison between RSI predictions and measured data for the ADP Fan 1 at approach conditions.

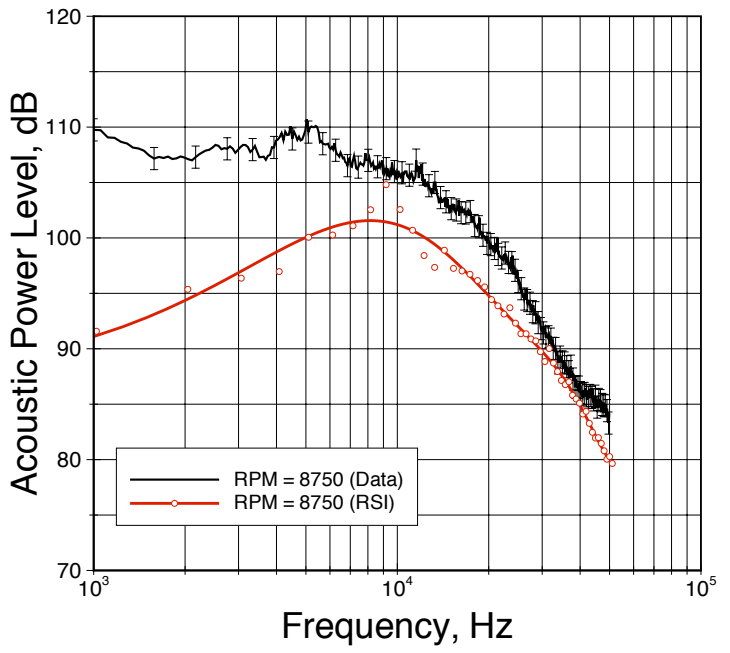

Figure 16. Narrow band acoustic power level comparison between RSI predictions and measured data for the ADP Fan 1 at take-off conditions.

This example illustrates the type of predictions achievable when using a higher fidelity code to make the predictions. The fan broadband noise predicted by RSI is compared to measured data from the model-scale ADP Fan 1 in Figure 15 for approach conditions and in Figure 16 for take-off conditions. The measured spectra, shown in black, have had their tonal content removed to emphasize the broadband noise comparison. In addition, the measured spectra have error bars for the $\pm 1 \mathrm{~dB}$ of uncertainty that was determined for the measured data. The predictions have similar spectral shapes compared to the measured data at frequencies higher than the peak frequency in the predictions. These predictions are based on a proper calibration of the background turbulence level in the CFD results. The predicted background turbulence levels were generally lower than measured levels and were raised for better agreement. With only this calibration, the CFD results were otherwise used as computed leading to the results shown in the figures. Since the measured noise comes from more sources than are included in the prediction model, the measured acoustic power levels are higher than the predicted acoustic power levels. ${ }^{16}$ 


\section{III.C. Statistical Tools}

Statistical codes attempt to make use of acoustic analogies or other theoretical formulations that describe the transfer of energy from turbulence to noise. Typically these codes require as input a Reynolds-Averaged Navier-Stokes (RANS) computational fluid dynamics (CFD) solution for the mean velocities, temperatures, turbulent kinetic energy, and perhaps dissipation. From models of how the space-time correlations of turbulent eddies are distributed in the plume, estimates of the noise produced from every segment of the plume are propagated through the inhomogeneous acoustic medium to the far field observer. These codes have the advantage that, to the extent local turbulent eddies can be considered independent noise sources and to the accuracy with which the statistically stationary turbulent flow can be calculated and the propagation computed, the flow can be as complicated as desired. However, the models relating turbulent kinetic energy to sound sources are not necessarily detailed enough. Some sources such as turbulent temperature fluctuation are typically ignored and the solution of the propagation is non-trivial for highly three-dimensional flows. Two statistical jet noise prediction codes called Jet3D and JeNo were evaluated. Fewer cases were run for these statistical prediction methods than were run for the semi-empirical ANOPP code above because these methods require substantial resources. The cases evaluated do span much of the parameter space as in the empirical evaluations, but much more sparsely.

\section{III.C.1. Statistical Jet Noise Prediction Tool - Jet3D}

Jet3D ${ }^{28}$ is based on a straightforward application of Lighthill's Acoustic Analogy in three dimensions, giving the far field mean-square acoustic pressure as a function of a spatial observer position and a time delay. The sources of sound are evaluated inside the source region at a retarded time and corresponding position. By formulating the jet noise problem into an equivalent linear acoustics problem, it becomes possible to handle complex three-dimensional flows and arbitrary nozzle configurations. Compared to other methods based on non-linear acoustic propagation and convected wave equation formulations, simplifying jet flow assumptions are not needed in this approach, and ray-tracing or flow-acoustic interaction models are not required. However, this method is dependent on source modeling, since the sources themselves produce analogous propagation effects through temporal and spatial phasing. While it has been difficult to obtain the appropriate flow field source information, the fidelity and quality of information available in a RANSbased CFD simulation has improved to the point where it is now possible to develop better models and make educated assumptions about any remaining unknowns. Refer to Hunter ${ }^{28}$ for details.

\section{III.C.2. Statistical Jet Noise Prediction Tool - JeNo}

The JeNo code ${ }^{29}$ is based on a derivation of the equations of motion after each flow variable is decomposed into its base flow and radiating components. These equations are split into two sets of equations that govern a non-radiating background flow plus its residuals. The residuals components are simply the difference between the original equations and their base-flow equivalents, and are arranged to form an acoustic analogy. The equivalent sources of sound are identified with the non-linear terms in the analogy. The source components are the usual Reynolds stresses and a fluctuating velocity/enthalpy term as a heat-related source component. In this modeling approach, the unsteady features of the flow are modeled, while the non-radiating base flow is taken to be the jet mean flow as calculated from a RANS flow solver.

Rather than assuming a quiescent acoustic medium, the mean flow effect is included by moving the linear component that includes the effect of the axial mean velocity gradient from the source side of the governing equations to the operator side. After combination, we obtain a third-order convective wave equation that is solved using Green's function techniques and source modeling. Version 1.0 of the JeNo code solves for the Green's function assuming mean flow axisymmetry and a locally parallel flow approximation. ${ }^{29}$

Source modeling is a crucial step in a physics-based prediction approach. It requires appropriate modeling of a two-point space-time velocity correlation. As shown in Khavaran and Bridges, ${ }^{30}$ the best representation of the experimental measurements are obtained when exponential functions are selected to represent both spatial and temporal dependencies of the correlation. Integration of the source correlation functions provide information on the source strength and spectral shape. The general shape of the radiated spectrum and its roll-off at the high and low ends of its frequency range depends on the source model as well as the accuracy of the the Green's function. Many details may be found in Khavaran et al. ${ }^{8,29,30}$ 

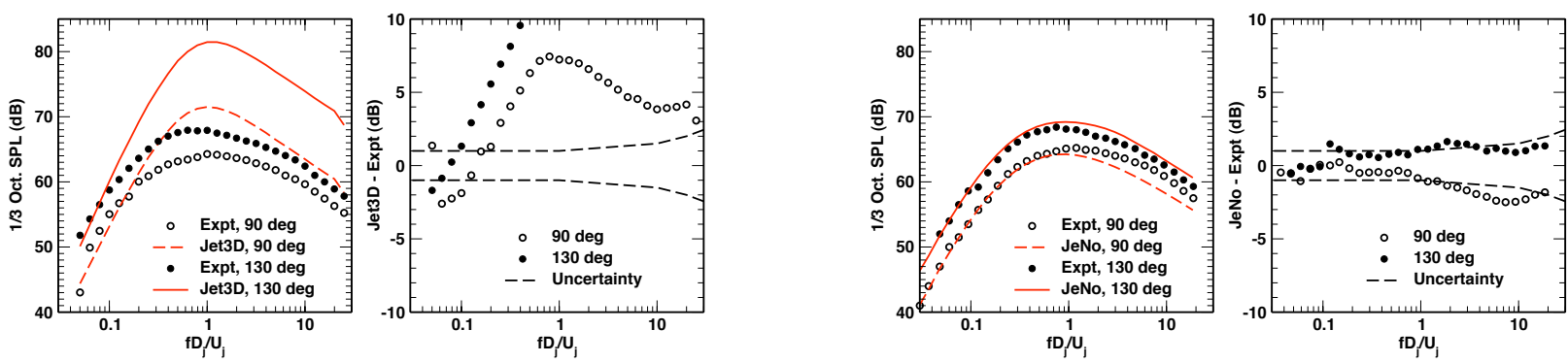

Figure 17. Comparison of statistical jet noise code predictions with measured data. Spectra at 90 deg and $130 \mathrm{deg}$ from the inlet axis (presented lossless at 100D) and the comparison error. Axisymmetric jet conditions: $M_{j}=0.51$, Temp. Ratio 0.955. Jet3D code results on the left. JeNo code results on the right.
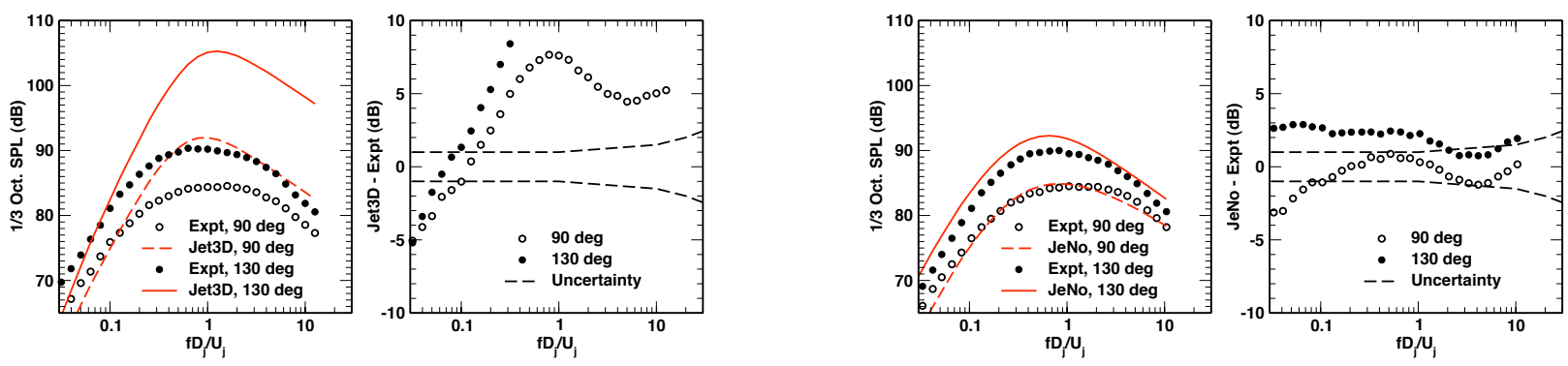

Figure 18. See caption for Figure 17. Axisymmetric jet conditions: $M_{j}=0.98$, Temp. Ratio $\mathbf{0 . 8 4 2}$.
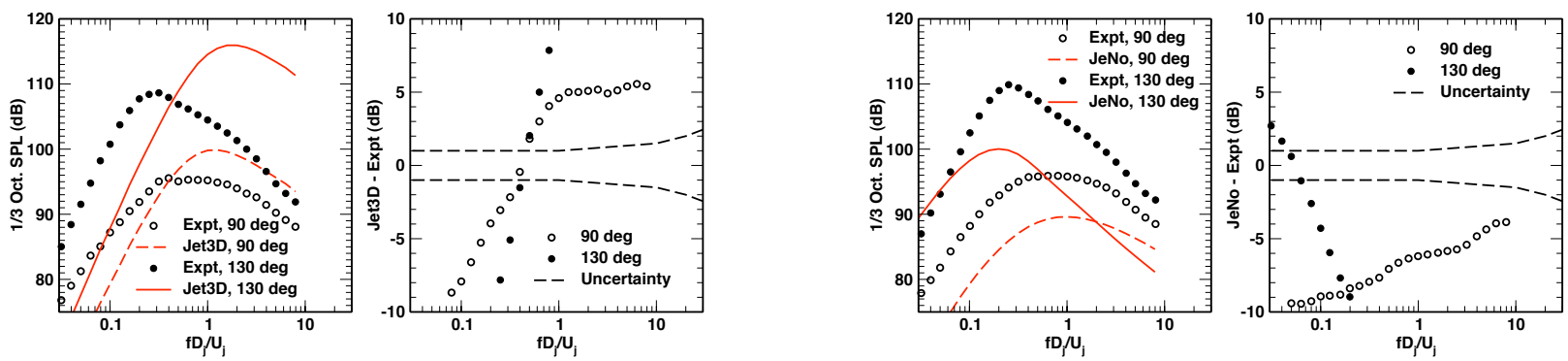

Figure 19. See caption for Figure 17. Axisymmetric jet conditions: $M_{j}=0.92$, Temp. Ratio 2.703.

\section{III.C.3. Sample Results}

All predictions were compared against experimental data from the NASA Glenn jet noise rigs. Far-field noise data were themselves validated against jet noise data from other high quality published data sets. The NASA facility also generated a large amount of flow field data that was required to validate the RANS solutions that serve as input for the statistical codes. The flow field data were validated against published flow data where available. The cross-facility comparisons were used, in conjunction with component analysis, to establish the experimental uncertainty of the data. More details may be found in Bridges et al. ${ }^{18}$

Sample results for the noise radiated from subsonic jets issuing from axisymmetric nozzles are shown next. Figure 17 shows jet noise predictions using the two different statistical codes compared to measured data. The Jet3D results on the left side show 1/3-octave spectral levels as a function of the Strouhal number. The two spectra are compared to measured data at two angles of directivity, $90 \mathrm{deg}$ and $130 \mathrm{deg}$ from the inlet, for a cold, axisymmetric jet with $M_{j}=0.51$ and static temperature ratio 0.955 . The figure next to it shows the comparison error between measured and predicted values along with an estimate of the uncertainty in the results. The same set of comparisons are shown on the right side for JeNo code predictions. Figure 18 shows the same type of data for a near sonic jet $M_{j}=0.98$ and static temperature ratio 0.842 and Figure 19 shows results for a hot jet $M_{j}=0.92$ and static temperature ratio 2.703 . The only good comparison between prediction and measurement is that for the cold, subsonic jet, JeNo predictions in Figures 17 and 18 where the error is within or near the margin of uncertainty across the spectrum. For hot jets, JeNo breaks down due to improper source modeling. The Jet3D results are clearly at variance with the single jet measurements. 
The calibration process associated with this $\operatorname{code}^{28}$ perhaps needs to be revisited in light of this newer data.

\section{III.D. Numerical Tools}

The highest fidelity tool for aircraft noise prediction is to numerically solve the fundamental equations of motion subject to the appropriate initial and boundary conditions. Such a code would ideally solve for the complete time-dependent turbulent flow and the radiated noise generated within or near a surface with complex geometry. However, the computer resources currently required for such a code are very large and the time to run is very long. For practicality, the scope of the problem must be reduced in order for the numerical tool to be useful. Two example code applications are presented here. One involves a hybrid approach for component level airframe noise prediction. A filtered set of flow equations to compute only the unsteady flow in a region where noise would be generated; then, computing the radiated noise using a separate method. The other application is to compute the propagation of sound using linearized flow equations in a well defined geometry like a duct.

\section{III.D.1. Airframe Noise Predictions using CFL3D}

The acoustic prediction of airframe noise using numerical techniques can be based on the acoustic analogy principles of aerodynamic sound and their extensions to solid surfaces in arbitrary motion. The noise associated with aerodynamic problems of interest can then be predicted, provided that the equivalent forces representing the sources of sound can be accurately modeled. Thus, the objective in this approach is to model the actual flow mechanisms that give rise to the sources of radiated sound. The simulation of these sources can be done by solving an appropriate set of fluid dynamics equations. While such methods cannot be considered practical prediction tools, they provide insights into the physical mechanisms of airframe noise generation that can be incorporated into models in the next generation of prediction tools.

Simulations have been performed using CFL3D, a CFD code developed at the NASA Langley Research Center. The CFL3D code uses a finite-volume formulation for the three-dimensional, time-dependent, thin layer Reynolds-Averaged Navier-Stokes (RANS) equations. The code employs a third-order upwind difference for the convection terms that is nominally second-order accurate on nonuniform grids. The viscous terms are discretized with second-order central differences. Time is advanced with a second-order backward difference. Further details may be found in Krist et al. ${ }^{31}$ and in Rumsey et al. ${ }^{32}$ When the flow simulations are completed, the near-field sources are then available to be used in an integral technique to compute the farfield signal. Here, the Ffowcs Williams-Hawkings (FW-H) equation solvers described by Lockard ${ }^{33}$ are used to predict the acoustic signature at various observer locations.

An example assessment of this numerical procedure is a comparison of simulation results with experimental data for a prototype airframe component interaction using tandem cylinders. The tandem cylinder CFL3D simulations were run with three different grids. The first grid had a block structure in the x-y plane perpendicular to the cylinders that was replicated in the spanwise z-direction along the cylinders. The total grid count for this grid covering a six diameter span was 80 million. This grid is denoted as Fxyz or the fine grid. Simulations based on a medium grid (derived from the finest grid by removing every other grid point in each direction) were performed to allow an assessment of the grid convergence of the computed solutions. This grid is denoted as Mxyz. In this case, the grid was expanded in the spanwise direction to 18 diameters, which is more representative of the experiment, and has a total of 30 million grid points. A third grid had the same spanwise resolution as the fine grid. This grid is denoted as MxyFz. Again, the spanwise extent of the grid covered 18 diameters, with 60 million grid points. A constant, non-dimensional time step was used for all simulations. The experimental results were obtained from the measurement of the radiated noise from the tandem cylinders in the QFF wind tunnel facility, at NASA Langley. Details of the computational and experimental efforts are given in Lockard et al. ${ }^{34}$

A comparison between the predictions using the three grids and the experimental results are shown using a narrow band analysis in Figure 20. All of the data have been scaled to an equivalent $1-\mathrm{Hz}$ bin width, so the presentation is in power spectral density. Both the experiment and predictions show a pronounced tone at low frequency, but the predicted frequency is slightly lower than that observed in the experiment. The Fxyz result was obtained by replicating the data from the span of 6 calculations, 3 times, to produce a span of 18, matching the length of the other calculations and the experiment. However, this produces an artificial spanwise correlation that produces slightly higher noise predictions. The elevated levels are also evident in the 1/3-octave results shown in Figure 21. Bars are included in the plot representing the 


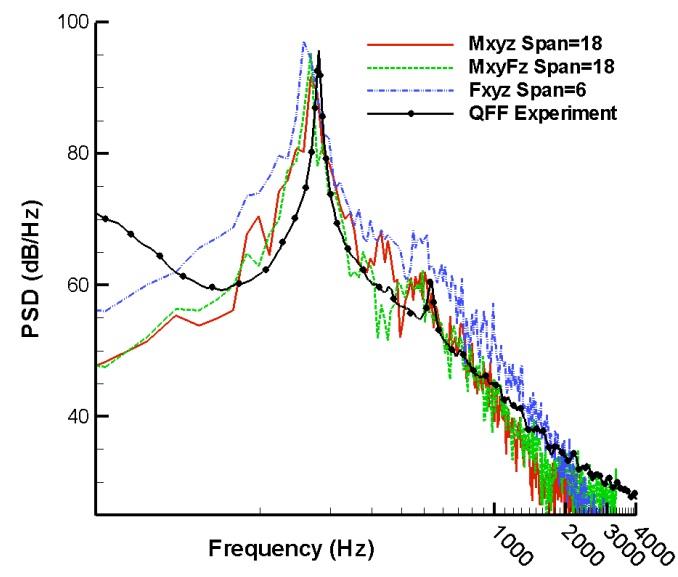

Figure 20. Power spectral density for both CFL3D predictions and measured data.

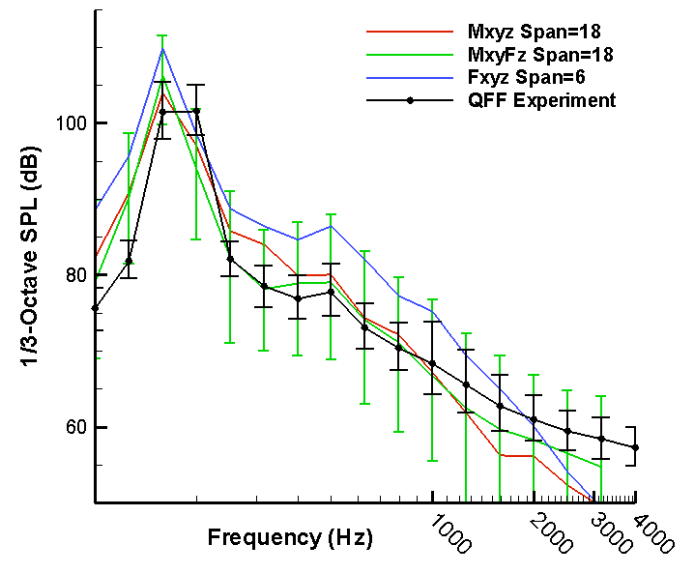

Figure 21. Comparison of $1 / 3$-octave spectra between CFL3D predictions and measured data.

scatter between runs in the experiment and between different averaging segments in the predictions. The time record available from the CFD was short, so only 5 segments of 4096 samples were used to obtain an average. The band shows the maximum and minimum values that were observed in the 5 segments. Although the numerical prediction shows large variations, even the experiment shows considerable scatter because of the highly intermittent nature of the shedding process. While numerical codes can capture the unique flow features around an airframe or parts of an airframe, the codes take a long time to run. Consequently, it is difficult to get good statistics to determine prediction uncertainty. It is desirable to have better precision than the approximately $\pm 10 \mathrm{~dB}$ level of uncertainty shown in the figure.

\section{III.D.2. Duct Acoustics and Liner Physics}

Noise prediction codes for aircraft nacelles have generally been divided into potential and rotational flow codes. Each code has advantages and disadvantages depending upon the problem being modeled, assumptions that are made, and computer facilities available to code users. ${ }^{35}$ Sample results from three of the codes listed in Table 1 compared to experimental data will be shown here. Two of the codes are potential flow type codes and the third is a rotational flow code.

1. Potential Flow Computer Codes - These codes solve the potential flow scalar wave equation coupled with the appropriate source, termination and wall impedance boundary conditions.

(a) Quasi-3D Code (labeled CH2DDS) - The quasi-3D approximation is applicable to duct geometries and flows for which the acoustic solution in one orthogonal direction is separable due to the fact that the sidewalls are both rigid and orthogonal to that direction. Quasi-3D acoustic analysis is restricted to a liner impedance that depends only on the axial coordinate and mean velocity fields that have only an axial component. Further, the axial velocity, mean pressure, and mean density are constant so that the uniform-flow scalar wave equation in terms of pressure and the acoustic pressure boundary conditions may be used. The solution is obtained by directly solving the matrix equation that results from using the Galerkin finite element method with the weighted residuals approximation technique. Details may be found in Watson et al. ${ }^{36}$

(b) 3D Code with Parabolic Approximation (labeled CH3DPA) - This duct fan noise propagation and radiation code has been developed that utilizes a parabolic approximation to the convected Helmholtz equation. ${ }^{10}$ This approach affords very efficient propagation calculations, thus allowing solutions for complex three-dimensional geometries to be handled with relatively low computational costs. This efficiency comes at the expense of a decrease in accuracy as the direction of propagation of an acoustic mode diverges from the preferred angle of the parabolic approximation and a loss of accuracy because reflections of acoustic waves in the axial direction are not captured in this formulation. 
2. Rotational Flow Computer Codes - These duct propagation models do not assume that the disturbance is irrotational.

(a) Quasi-3D Code (labeled LEE2DDS) - The quasi-3D code is based upon a finite element simulation of the linearized equations that govern conservation of mass and momentum for the fluid in the duct. This rotational flow code is applicable to duct geometries and flows for which the solution in one orthogonal direction (assumed to be in the plane of the duct cross-section) is separable, due to the fact that the sidewalls are both rigid and orthogonal to that direction. Quasi-3D analysis is valid only if the mean velocity field, does not have a component in the separable dimension and the other mean flow quantities are also independent of that dimension For the solution to be separable (as required in quasi-3D) the wall impedance must also be a function of the axial coordinate only. More technical details concerning the quasi-3D code is presented in Watson et al. ${ }^{37}$

The duct acoustic codes are used to compute the attenuation of sound propagating in a duct due to the presence of acoustic absorbing material along the duct wall. Usually, the exact values expected for the attenuation are not available. Predicted attenuations are therefore compared to measured data. Additionally, because the code inputs must be measured, they are not known exactly but are subject to measurement uncertainty. Uncertainty also exists in the measured attenuation. Therefore, measurement and predictions are compared on a statistical basis, and uncertainty bounds are placed on both quantities.

These codes are also applied as a data reduction tool to educe the wall impedance from measured data. Using these codes for this purpose allows the measurement method to be non-invasive whether the sound is at normal incidence ${ }^{38}$ or in a grazing flow environment. ${ }^{39}$ The duct codes model the propagating acoustic field and mediate between the complex acoustic pressures, measured away from the test sample surface, and the surface impedance of the sample. The advantage of this method is that a global determination of impedance is achieved. A disadvantage is that a high level of field model fidelity is required to link the pressure measurements to the liner impedance. A lack of field model fidelity decreases the quality of the impedance measurement.

For the wall acoustic treatment of interest here, the focus is on a treatment with a perforate-overhoneycomb (POHC) construction, a single layer liner, and on the two key aspects of POHC liner absorption. One aspect is the dissipation process that controls the absorption level and bandwidth, and the other is the combined acoustic reactance of the honeycomb cell and perforate that determines the resonant frequencies. Each of these aspects is captured by a single parameter, the acoustic impedance. It is this parameter that links the liner physics to the duct airway propagation model via a boundary condition on the duct airway propagation process. Models for POHC liners are semi-empirical.

The sample results from these parts of this assessment illustrate an application of a Monte-Carlo approach to estimating the uncertainties for both the impedance predictions and measurements and the duct sound attenuation predictions and measurements. The predictions were based on thirty-one simulations where each input parameter was randomly selected from its respective Gaussian distribution. The means and standard deviations of the appropriate output values were determined and then used to compute the $95 \%$ confidence intervals. On the measurement side, between eight and fourteen tests were conducted in order to allow for a small-sample statistical evaluation. ${ }^{14}$ The measurement $95 \%$ confidence intervals were then compared to the predicted $95 \%$ confidence intervals. The complete details for this process are found for the duct acoustics codes assessment in Nark et al. ${ }^{40}$ and for the liner impedance modeling assessment in Jones et al. ${ }^{41}$

Sample results are shown in Figures 22 and 23 where measurements and predictions are shown for duct attenuation (bottom plot) and the corresponding resistance (top plot) of the wall treatment in the duct. The duct attenuation predictions were made by the three sample codes described above. The sample resistance predictions were made by the Two-Parameter impedance model listed in Table 1. In the resistance plots, the blue lines represent the $95 \%$ confidence interval (also referred to as the uncertainty range or simply the confidence interval) for the predictions and the red bars represent the $95 \%$ confidence intervals for the measurements. The duct attenuation plots show a high and a low symbol to represent the confidence interval. For example, the confidence intervals were computed from duct attenuation measurements at each frequency and displayed by green squares on the plot. The separation between the two green squares at a particular frequency represents the uncertainty range in the measured attenuation. The confidence intervals for measurements and predictions are provided at each frequency of interest in each figure. These confidence intervals in both the resistance plot and the attenuation plot indicate that if another simulation 


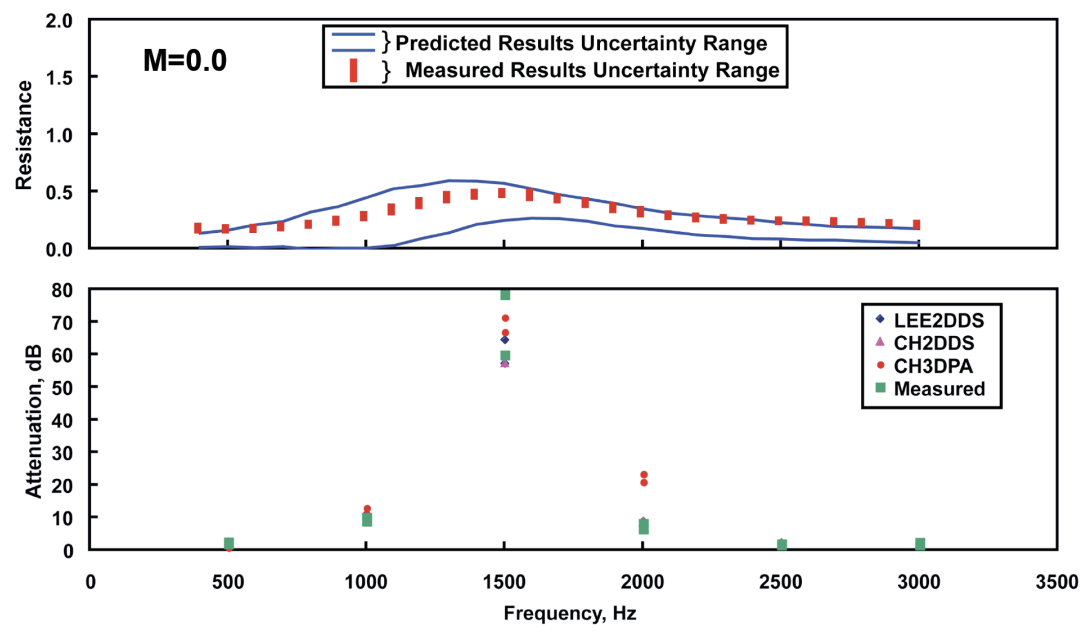

Figure 22. Comparison of measured and predicted confidence intervals for a typical single-layer perforate-overhoneycomb liner. Top figure shows the liner resistance. Bottom figure is the attenuation of sound in a duct with the above liner resistance. Impedance measured at normal incidence. Duct flow: $M=0.0$.

was conducted, there is a $95 \%$ confidence that the resulting prediction would fall within these limits. As noted in the figures, the results are shown for a duct with no flow, Figure 22, and for a duct with a mean flow of $M=0.3$, Figure 23. In general, the predicted duct attenuation mean values of the simulations are observed to track the mean values of the measured attenuation data quite well. At no mean flow, enormous attenuations (60 to $80 \mathrm{~dB}$ ) are observed at or near the resonance frequency. These attenuations decrease with the addition of a mean flow in the duct. At frequencies away from resonance, measured attenuations are less than those at resonance.

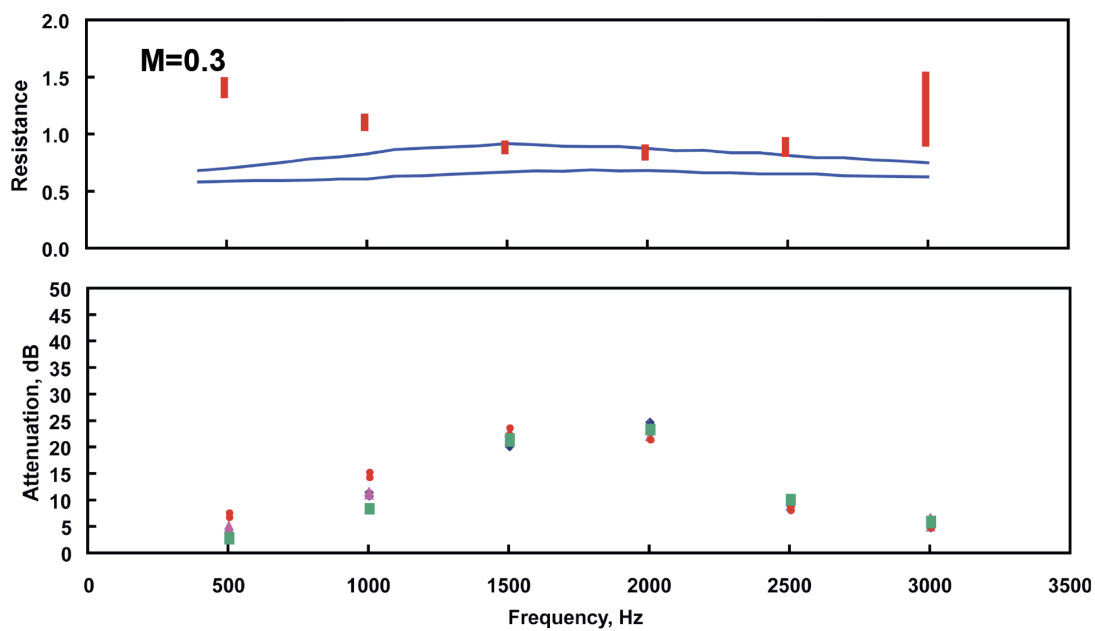

Figure 23. See caption and legend for Figure 22. Impedance measured at grazing incidence. Duct flow: $M=0.3$.

The plot of resistance in Figure 22 shows results for no flow where the sound is at normal incidence to the treatment surface. The mean of the measured resistance increases with frequency from a low value at the lower frequencies to a high value near $1500 \mathrm{~Hz}$, the approximate resonant frequency of the treatment. A comparison of the predicted and measured resistances reveal that the prediction exhibits a broad peak in the mean resistance in the vicinity of resonance, the prediction shows a significant growth in its confidence intervals through the region of increasing resistance associated with resonance, and the prediction fails to track the measured confidence intervals throughout the whole frequency range. The predicted mean resistance is generally lower than measured. 
Figure 23 contains results for the same test liner with a grazing flow average Mach number of 0.3 . With the addition of flow, there is a general concave upward trend relative to the no flow case and a large confidence interval at $3000 \mathrm{~Hz}$. This large confidence interval may be due to violating the assumptions made about the acoustic modes in the duct when implementing the current measurement process. The predicted resistance confidence interval spectrum for the model encompasses only two of the measured confidence intervals (at 1500 and $2000 \mathrm{~Hz}$ ), although the measured confidence interval at $2500 \mathrm{~Hz}$ is barely above the upper limit of the predicted confidence interval spectrum. In summary, the resistance means increase significantly with the increase of mean flow. This is indicative of liner nonlinearity. The model represented here produces resistances that are at or near the measured resistances near resonance (around $1500 \mathrm{~Hz}$ with no flow; shifts upward with increased mean flow), and under predicts the measured resistances at frequencies away from resonance.

\section{Concluding Remarks}

The NASA Fundamental Aeronautics Program has set forth the challenge of developing an MDAO capability that is both accurate and flexible in its fidelity to enable the exploration of design spaces beyond those occupied by current aircraft designs. To achieve this goal, and to have confidence in the results obtained, the computational tools involved in MDAO must simulate the underlying physical process within a quantifiable level of accuracy. Both computational and experimental efforts are required to meet this challenge. As computational modeling and the ability to make predictions advances, experimental data are required both for guiding the modeling effort and for validation. This paper outlined the process NASA is using to assess its current capability to predict aircraft noise and establish a baseline for progress at the outset of developing the aircraft noise prediction portion of this MDAO capability vision.

The range of aircraft noise prediction tools encompassed semi-empirical, analytical, statistical, and numerical computer codes. Results from these codes were compared to experimental data and the level of difference (or error) was quantified. Also in keeping with the assessment process and to the extent possible, the levels of uncertainty involved in both the computational process and the experimental data gathering process were quantified. This was done to add credibility to the assessment since it would then be possible to state a goodness or badness of a prediction by using a quantified number. Using the contributions of many researchers involved with the assessment, samples were shown of the types of results obtained. These included noise predictions for aircraft components using modules from the semi-empirical ANOPP code such as fan noise, jet noise, and airframe noise from flaps. A combination of these modules to simulate an engine system showed the results predicted for combined sources and integrated noise levels. This was also done to make a prediction for the noise radiated from a complete aircraft. Higher fidelity codes were then used for predictions of fan and jet noise. For jet noise predictions, the differences were highlighted between two CFD-based, statistical source model codes. The sources of noise in a tandem cylinder problem were computed using unsteady CFD and the radiated noise was computed separately. Finally, an example was shown for the results from computing the uncertainty statistics for both the prediction and measured data using codes to predict the attenuation of sound in a lined duct and models to predict the impedance of the duct lining. It is intended that all the results and analysis of this assessment will be documented in a forthcoming NASA Technical Publication.

\section{References}

\footnotetext{
${ }^{1}$ Oberkampf, W. L. and Trucano, T. G., "Verification and Validation in Computational Fluid Dynamics," Prog. Aero. Sci., Vol. 38, 2002, pp. 209-272.

2Zorumski, W. E., "Aircraft Noise Prediction Program Theoretical Manual, Parts 1 \& 2," NASA TM-83199, February 1982, (Revision Available).

${ }^{3}$ Raney, J. P., "Development of a New Computer System for Aircraft Noise Prediction," AIAA Paper No. 75-536, 1975.

${ }^{4}$ Raney, J. P., "Noise Prediction Technology for CTOL Aircraft," NASA TM-78700, May 1978.

${ }^{5}$ Ventres, C. S., Theobald, M. A., and Mark, W. D., "Turbofan Noise Generation: Volume 1, Analysis," NASA CR-167952, July 1982.

${ }^{6}$ Meyer, H. D. and Envia, E., "Aeroacoustic Analysis of Turbofan Noise Generation," NASA CR-4715, March 1996. 678 .

${ }^{7}$ Nallasamy, M. and Envia, E., "Computation of Rotor Wake Turbulence Noise," J. Sound Vib., Vol. 282, 2005, pp. 649-

${ }^{8}$ Khavaran, A. and Kenzakowski, D. C., "Noise Generation in Hot Jets," AIAA Paper No. 2007-3640, 2007.
} 
${ }^{9}$ Tam, C. K. W., "Computational Aeroacoustics: An Overview of Computational Challenges and Applications," Int. J. Comp. Fluid Dyn., Vol. 18, No. 6, 2004, pp. 547-567.

${ }^{10}$ Nark, D. M., Farassat, F., Pope, D. S., and Vatsa, V., "The Development of the Ducted Fan Noise Propagation and Radiation Code CDUCT-LARC," AIAA Paper No. 2003-3242, 2003.

${ }^{11}$ Lele, S. K., "Computational Aeroacoustics: A Review," AIAA Paper No. 97-0018, 1997.

${ }^{12}$ Stern, F., Wilson, R. V., Coleman, H. W., and Paterson, E. G., "Comprehensive Approach to Verification and Validation of CFD Simulations-Part1: Methodolgy and Procedures," J. Fluids Eng., Vol. 123, 2001, pp. 793-802.

${ }^{13}$ AIAA, "Guide for the Verification and Validation of Computational Fluid Dynamic Simulations," AIAA-G-077-1998, 1998.

${ }^{14}$ Coleman, H. W. and Steele, W. G., Experimentation and Uncertainty Analysis for Engineers, 2nd Edition, John Wiley \& Sons, Inc., New York, NY, 1999.

${ }^{15}$ Taylor, J. R., An Introduction to Error Analysis: The Study of Uncertainties in Physical Measurements, University Science Books, Sausalito, CA, 2nd ed., 1997.

${ }^{16}$ Envia, E., Tweedt, D., Elliott, D., Fite, E., Hughes, C., Podboy, G., Sutliff, D., and Woodward, R., "An Assessment of Current Fan Noise Prediction Capability," AIAA Paper No. 2008-2991, 2008.

${ }^{17}$ Stone, J. R., Krejsa, E. A., and Clark, B. J., "Semi-Empirical Model for Coannular Nozzle Component Noise Extraction and Correlation Including the Effects of Noise Reduction Devices," AIAA Paper No. 2003-1060, 2003.

${ }^{18}$ Bridges, J. E., Khavaran, A., and Hunter, C., "Assessment of Current Jet Noise Prediction Capabilities," AIAA Paper No. 2008-2933, 2008.

${ }^{19}$ Emmerling, J. J., Kazin, S. B., and Matta, R. K., "Core Engine Noise Program, Volume III, Supplement 1 - Prediction Method," FAA RD-74-125, March 1976.

${ }^{20}$ Hough, J. W. and Weir, D. S., "Small Engine Technology (SET) - Task 13 ANOPP Noise Prediction for Small Engines: Jet, Core, and Turbine Module Revisions," Tech. Rep. No. 21-9655, AlliedSignal Engines, Phoenix, AZ, April 1997.

${ }^{21}$ Krejsa, E. A. and Valerino, M. F., "Interim Prediction Method for Turbine Noise," NASA TM-X-73566, November 1976.

${ }^{22}$ Weir, D. S., editor, Engine Validation of Noise and Emission Reduction Technology Phase 1 (RASER) Task 8 - Final Report, NASA CR-2008-215225, 2008.

${ }^{23}$ Weir, D. S. and Mendoza, J., "Baseline Noise Measurements from the Engine Validation of Noise and Emissions Program," AIAA Paper No. 2008-2807, 2008.

${ }^{24}$ Brooks, T. F. and Humphreys, W. H., "Flap-Edge Aeroacoustic Measurements and Predictions," J. Sound Vib., Vol. 261, 2003, pp. 31-74.

${ }^{25}$ Khorrami, M., Lockard, D., Humphreys, W., Choudhari, M., and Van de Ven, T., "Preliminary Analysis of Acoustic Measurements from NASA-Gulfstream Airframe Noise Flight Test," AIAA Paper No. 2008-2814, 2008.

${ }^{26}$ Fink, M. R., "Airframe Noise Prediction Method," FAA RD-77-29, March 1977.

${ }^{27}$ Fink, M. R., "Noise Component Method for Airframe Noise," J. Aircraft, Vol. 16, No. 10, 1979, pp. 659-665.

${ }^{28}$ Hunter, C. A., An Approximate Jet Noise Prediction Method Based on Reynolds-Averaged Navier-Stokes Computational Fluid Dynamics Simulation, D.Sc. thesis, The George Washington University, January 2002.

${ }^{29}$ Khavaran, A., Bridges, J. E., and Georgiadis, N., "Prediction of Turbulence-Generated Noise in Unheated Jets, Part 1: JeNo Technical Manual (Version 1.0)," NASA TM-2005-213827, July 2005.

${ }^{30}$ Khavaran, A. and Bridges, J. E., "Modelling of Fine-Scale Turbulence Mixing Noise," J. Sound Vib., Vol. 279, 2005, pp. 1131-1154.

${ }^{31}$ Krist, S. L., Biedron, R. T., and Rumsey, C. L., "CFL3D User's Manual (Version 5.0)," NASA TM-1998-208444, June 1998.

${ }^{32}$ Rumsey, C. L., Biedron, R. T., and Thomas, J. L., "CFL3D: Its History and Some Recent Applications," NASA TM112861, May 1997.

${ }^{33}$ Lockard, D. P., "A Comparison of Ffowcs Williams-Hawkings Solvers for Airframe Noise Applications," AIAA Paper No. 2002-2580, 2002.

${ }^{34}$ Lockard, D. P., Khorrami, M. R., Choudhari, M. M., Hutcheson, F. V., and Brooks, T. F., "Tandem Cylinder Noise Predictions," AIAA Paper No. 2007-3450, 2007.

${ }^{35}$ Watson, W. R., Nark, D. M., Jones, M. G., and Parrott, T. L., "Assessment of 3-D Codes for Predicting Liner Attenuation in Flow Ducts," AIAA Paper No. 2008-2828, 2008.

${ }^{36}$ Watson, W. R., Jones, M. G., and Parrott, T. L., "A Quasi-3D Theory for Impedance Eduction in Uniform Grazing Flow," AIAA Paper No. 2005-2848, 2005.

${ }^{37}$ Watson, W. R., Tracy, M. B., Jones, M. G., and Parrott, T. L., "Impedance Eduction in the Presence of Shear Flow," AIAA Paper No. 2001-2263, 2001.

${ }^{38}$ Jones, M. G. and Stiede, P. E., "Comparison of Methods fro Determining Specific Acoustic Impedance," J. Acoust. Soc. Am., Vol. 101, No. 5, 1997, pp. 2694-2704.

${ }^{39}$ Jones, M. G., Watson, W. R., Tracy, M. B., and Parrott, T. L., "Comparison of Two Acoustic Waveguide Methods for Determining Liner Impedance," AIAA Paper No. 2001-2266, 2001.

${ }^{40}$ Nark, D. M., Watson, W. R., and Jones, M. G., "Uncertainty and Sensitivity Analyses of Duct Propagation Models," AIAA Paper No. 2008-2832, 2008.

${ }^{41}$ Jones, M. G., Parrott, T. L., and Watson, W. R., "Uncertainty and Sensitivity Analyses of a Two-Parameter Impedance Prediction Model," AIAA Paper No. 2008-2928, 2008. 


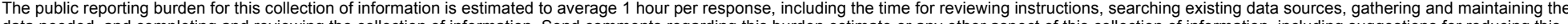

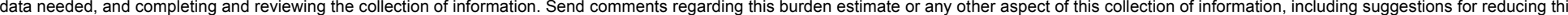

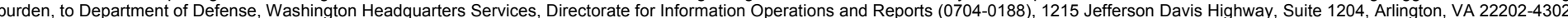

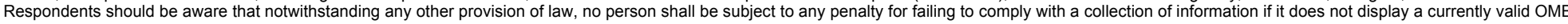
control number.

PLEASE DO NOT RETURN YOUR FORM TO THE ABOVE ADDRESS.

\section{REPORT DATE $(D D-M M-Y Y Y Y)$ \\ 2. REPORT TYPE \\ 3. DATES COVERED (From - To)}

01-07-2008

Technical Memorandum

\section{TITLE AND SUBTITLE}

Process for Assessing NASA's Capability in Aircraft Noise Prediction Technology

\section{5a. CONTRACT NUMBER}

5b. GRANT NUMBER

5c. PROGRAM ELEMENT NUMBER

6. AUTHOR(S)

Dahl, Milo, D.

\section{5d. PROJECT NUMBER}

5e. TASK NUMBER

5f. WORK UNIT NUMBER

WBS 984754.02.07.03.17.02

\section{PERFORMING ORGANIZATION}

REPORT NUMBER

E-16538

National Aeronautics and Space Administration

John H. Glenn Research Center at Lewis Field

Cleveland, Ohio 44135-3191

9. SPONSORING/MONITORING AGENCY NAME(S) AND ADDRESS(ES)

National Aeronautics and Space Administration

Washington, DC 20546-0001

\section{DISTRIBUTION/AVAILABILITY STATEMENT}

Unclassified-Unlimited

Subject Category: 71

Available electronically at http://gltrs.grc.nasa.gov

This publication is available from the NASA Center for AeroSpace Information, 301-621-0390

\section{SPONSORING/MONITORS ACRONYM(S) \\ NASA; AIAA \\ 11. SPONSORING/MONITORING REPORT NUMBER \\ NASA/TM-2008-215268; AIAA-2008- 2813}

\section{SUPPLEMENTARY NOTES}

\section{ABSTRACT}

An acoustic assessment is being conducted by NASA that has been designed to assess the current state of the art in NASA's capability to predict aircraft related noise and to establish baselines for gauging future progress in the field. The process for determining NASA's current capabilities includes quantifying the differences between noise predictions and measurements of noise from experimental tests. The computed noise predictions are being obtained from semi-empirical, analytical, statistical, and numerical codes. In addition, errors and uncertainties are being identified and quantified both in the predictions and in the measured data to further enhance the credibility of the assessment. The content of this paper contains preliminary results, since the assessment project has not been fully completed, based on the contributions of many researchers and shows a select sample of the types of results obtained regarding the prediction of aircraft noise at both the system and component levels. The system level results are for engines and aircraft. The component level results are for fan broadband noise, for jet noise from a variety of nozzles, and for airframe noise from flaps and landing gear parts. There are also sample results for sound attenuation in lined ducts with flow and the behavior of acoustic lining in ducts.

\section{SUBJECT TERMS}

Noise prediction; Aircraft noise; Engine noise; Computer simulation; Errors; Sensitivity analysis; Statistical analysis; Accuracy;

Acoustic ducts; Acoustic attenuation

\begin{tabular}{|c|c|c|c|}
\hline \multicolumn{3}{|c|}{ 16. SECURITY CLASSIFICATION OF: } & \multirow{2}{*}{$\begin{array}{l}\text { 17. LIMITATION OF } \\
\text { ABSTRACT } \\
\text { UU }\end{array}$} \\
\hline $\begin{array}{l}\text { a. REPORT } \\
\text { U }\end{array}$ & $\begin{array}{l}\text { b. ABSTRACT } \\
\mathrm{U}\end{array}$ & $\begin{array}{l}\text { c. THIS } \\
\text { PAGE } \\
\text { U }\end{array}$ & \\
\hline
\end{tabular}

18. NUMBER
OF
PAGES
33

19a. NAME OF RESPONSIBLE PERSON
STI Help Desk (email:help@sti.nasa.gov)
19b. TELEPHONE NUMBER (include area code)
301-621-0390



